\title{
The genesis of Typhoon Nuri as observed during the Tropical Cyclone Structure 2008 (TCS-08) field experiment - Part 3: Dynamics of low-level spin-up during the genesis
}

\author{
L. L. Lussier III ${ }^{1}$, M. T. Montgomery ${ }^{1}$, and M. M. Bell ${ }^{2}$ \\ ${ }^{1}$ Naval Postgraduate School, Monterey, California, USA \\ ${ }^{2}$ University of Hawaii at Manoa, Honolulu, Hawaii, USA \\ Correspondence to: L. L. Lussier III (lussier@ucar.edu) \\ Received: 10 April 2013 - Published in Atmos. Chem. Phys. Discuss.: 15 October 2013 \\ Revised: 6 June 2014 - Accepted: 25 June 2014 - Published: 27 August 2014
}

\begin{abstract}
Aircraft reconnaissance data collected during the Tropical Cyclone Structure 2008 field campaign are used to examine further kinematical, dynamical, and thermodynamical aspects of the genesis of Typhoon Nuri. Data from the first two missions into the pre-Nuri disturbance document the transition from a tropical wave to a tropical depression. Dropwindsonde-derived tangential wind profiles at several radii from the low-level circulation center indicate that the magnitude of low-level circulation increases and that the corresponding tangential velocity maximum moves inward from the first to second reconnaissance mission. To compliment these findings, a three-dimensional variational analysis incorporating both dropwindsonde and aircraft Doppler radar data is conducted. These data are used to perform circulation tendency calculations at multiple distances from the lowlevel circulation center. The results demonstrate a net spinup of the system-scale circulation in the low levels near the center and in the outer regions of the recirculating Kelvin cat's eye circulation. In these regions, the spin-up tendency due to the influx of cyclonic absolute vorticity exceeds the frictional spin-down tendency for both Nuri missions. The system-scale spin-up is found to be accompanied by areas of low-level vorticity concentration through vortex-tube stretching associated with cumulus convection. The areal coverage and intensity of these high-vorticity regions increase between the first and second Nuri missions.

The findings of this study are consistent in some respects to the Nuri observational analysis carried out by Raymond and López-Carrillo (2011), but differ in their suggested key results and related scientific implications that the pre-
\end{abstract}

Nuri disturbance was spinning down in the planetary boundary layer on the first day of observations. The findings herein strongly support a recent tropical cyclogenesis model positing that the Kelvin cat's eye circulation of the parent wavelike disturbance provides a favorable environment for convective vorticity organization and low-level spin-up on the mesoscale.

\section{Introduction}

Although advances in the understanding and prediction of some aspects of mature tropical cyclones have improved over recent years (AMS Statement, 2007), there remain significant challenges to fully understand the problem of tropical cyclogenesis. Two significant hurdles that must be overcome are (i) gaining a complete understanding of the complex multi-scale interactions that occur during the transition of a synoptic-scale precursor to a mesoscale vortex and (ii) addressing the lack of in situ observations in areas where seedling disturbances for tropical cyclones originate and intensify.

In their observational study, Dunkerton et al. (2009, hereafter DMW09) developed a new tropical cyclogenesis model that outlines the dynamics and thermodynamics of the genesis sequence on the synoptic, meso-, and convective scales. This model was presented for disturbances originating from African easterly waves or other tropical waves and related hydrodynamic instabilities in a mean shear flow. Using three independent data sets, European Centre for Medium-Range 
Weather Forecasts Reanalysis data (ECMWF ERA-40), Tropical Rainfall Measurement Mission (TRMM) 3B42 3hourly precipitation and best-track data from the National Hurricane Center (NHC), the Kelvin cat's eye within the critical layer of a tropical easterly wave was shown to be the preferred region of storm formation, more precisely located near the intersection of the wave critical latitude and trough axis. The wave critical layer is the region surrounding a nonlinear wave critical latitude or surface in latitudinal shear flow where the wave's phase speed equals the mean flow velocity. In the enclosed Kelvin cat's eye, particles are trapped and recirculate, rather than being swept one way or the other by the surrounding latitudinal shear. DMW09 articulated, in the form of scientific hypotheses, three main ways that the recirculating cat's eye region of a tropical wave provide a favorable environment for tropical cyclogenesis: (H1) wave breaking or roll-up of the cyclonic vorticity and moisture near the critical surface in the lower troposphere provides the moist vorticity seedlings and a favorable environment for vorticity aggregation leading to tropical cyclone formation; (H2) the cat's eye is a region of quasi-closed Lagrangian circulation, and air is repeatedly moistened by convection and protected to some degree from dry air intrusion, which favors a predominantly convective type of heating profile; (H3) the parent wave is maintained and possibly enhanced by diabatically amplified mesoscale vortices within the cat's eye. The combination of the foregoing hypotheses $\mathrm{H} 1-\mathrm{H} 3$ was labeled the marsupial paradigm by DMW09 insofar as the cyclogenesis sequence is likened to the development of a marsupial infant in its mother's pouch. The "juvenile" proto-vortex is carried along and protected by the "mother" wave until it is strengthened into a self-sustaining entity. The cat's eye within the wave critical layer is thus dubbed the "wave's pouch" or simply, "pouch".

Data collected during two recent field campaigns have provided in situ observations to test the foregoing hypotheses under various atmospheric conditions and in different tropical cyclone basins. The THORPEX (The Observing System Research and Predictability Experiment) Pacific Regional Campaign (TPARC) and Tropical Cyclone Structure 2008 (TCS-08) experiment took place in the western North Pacific basin in August and September of 2008 (Elsberry and Harr, 2008). Data collected from these campaigns provided the first opportunity to test the applicability of the new tropical cyclogenesis model in the western North Pacific basin for the case of westward propagating disturbances. The PreDepression Investigation of Cloud systems in the Tropics (PREDICT) campaign took place in the Atlantic basin in 2010 (Montgomery et al., 2012). This campaign focused exclusively on collecting data in the pre-formation stage of potential tropical cyclones. The tropical cyclogenesis cases of Karl, Matthew, and Gaston have been examined in the context of this new tropical cyclone model (Smith and Montgomery, 2012; Rutherford and Montgomery, 2012; Wang,
2012). Analyses of data from these two campaigns have provided new insight into the tropical cyclogenesis problem.

The case of Typhoon Nuri was one of the more robustly sampled disturbances among these campaigns as aircraft reconnaissance missions flew through the disturbance on four consecutive days, allowing observation of the storm during the tropical wave, tropical depression, tropical storm, and typhoon stages. Montgomery et al. (2010a) used observations and global model analyses to examine the formation of Typhoon Nuri on the synoptic and meso- $\alpha$ scales. They showed that the synoptic-scale precursor disturbance was a tropical easterly wave with maximum amplitude in the lower troposphere ( $850 \mathrm{hPa}$ and below). The characteristics of the wave including propagation speed and direction, low-level vorticity maximum, and distribution of precipitation were shown to be consistent with previous observational studies of easterly waves in the western North Pacific basin dating back to the 1970s (e.g., Chang, 1970; Reed and Recker, 1971). The wave-like characteristics of this disturbance provided the opportunity for Montgomery et al. (2010a) to test the applicability of the marsupial paradigm in the western North Pacific basin. Montgomery et al. (2010a) showed that a tropical wave in the western North Pacific basin has the ability to form a protective wave pouch that is more moist than the surrounding environment. Additionally, they showed that Typhoon Nuri's wave pouch was favorable for convection and that the "sweet spot" proved to be the favored region for lowlevel vorticity aggregation and growth of the proto-vortex.

While Montgomery et al. (2010a) provided an overview of the tropical cyclogenesis sequence on larger scales, they did not address mesoscale organization within the wave pouch. A complimentary study by Raymond and López-Carrillo (2011, hereafter RL11) used dropwindsonde observations and airborne Doppler radar retrievals to examine the circulation budget of Typhoon Nuri. The analyses of RL11 showed that for the first research flight (hereafter Nuri 1), there is an absolute circulation maximum from the surface to $4 \mathrm{~km}$ that is nearly constant in magnitude with height. Contrarily, RL11 show that the vertical profile of absolute circulation in the second Nuri flight (hereafter Nuri 2) revealed a distinct maximum at the $5 \mathrm{~km}$ level. By calculating the circulation tendency over an area roughly enclosed by the dropwindsonde locations for each mission, RL11 concluded that there was a net spin-down of the vortex in the planetary boundary layer (PBL) during Nuri 1 on account of surface friction dominating the spin-up tendency associated with the influx of cyclonic absolute vorticity into the analysis region.

RL11 and Raymond et al. (2011) reasoned that since Nuri 1 was spinning down in the lower levels, a thermodynamic mechanism was required to reinitiate low-level spinup. The basis for this hypothesis can be traced back to a set of cumulus ensemble numerical experiments in a twodimensional, non-rotating equatorial-like environment performed by Raymond and Sessions (2007). In that study, the authors found that increased stabilization of the atmosphere 
(through the generation of a mid-level warm temperature perturbation coupled with the generation of a cold temperature perturbation in the lower troposphere) resulted in a concentrated inflow in a shallow layer in the lower troposphere. They found also that the thermal stabilization was more effective than the moistening of a column in fostering low-level convergence. The implication is that if realistic values of ambient rotation associated with a tropical wave or monsoon trough were included, this convergence would import sufficient absolute vorticity to overcome that lost to the surface by friction. This thermodynamic configuration was argued to be an essential ingredient in the spin-up of a tropical cyclone by altering the vertical mass flux ${ }^{1}$ profile and suppressing the lateral export of moist entropy (Raymond et al., 2011). In the case of Nuri 1, RL11 showed that the area-averaged vertical mass flux maximum was located near $10 \mathrm{~km}$, while the lower levels (below $4 \mathrm{~km}$ ) exhibited a near-zero vertical mass flux profile with a local region of negative values, presumably in association with mesoscale convective downdrafts. In Nuri 2, the vertical mass flux maximum was found near $5 \mathrm{~km}$. They postulated that as the height of the vertical mass flux maximum is lowered, the low-level convergence would be enhanced and thus, the processes described above would increase the low-level vorticity.

While RL11 and Raymond et al. (2011) offer a useful and insightful compliment to the large-scale overview presented in Montgomery et al. (2010a), there remain fundamental questions regarding the mesoscale organization of Typhoon Nuri that need to be addressed. Montgomery and Smith (2012) first questioned the conclusions presented in RL11 and Raymond et al. (2011). Montgomery and Smith (2011) used dropwindsonde data from the Nuri missions to examine the thermodynamic characteristics of the recirculating pouch region of the wave-like disturbance in which Nuri formed. They showed that, on the scale of the pouch region, the cooling in the lower troposphere between Nuri 1 and 2 was negligible and the warming in the upper troposphere was less than $1 \mathrm{~K}$, raising significant questions concerning the necessity of the thermodynamic control postulated by Raymond et al. (2011). Along these lines, Wang (2012) suggested that the evolution of circulation dynamics and thermal stabilization differ on different spatial scales within the wave pouch. In the inner region, where convective processes dominate, Wang (2012) showed that low-level spin-up occurs and thermal stabilization is not present. Additionally, Wang (2012) showed that the outer pouch region is characterized by weak thermal stabilization and is accompanied by low-level spin-down.

\footnotetext{
${ }^{1}$ We note that the vertical mass flux presented in RL11 is actually an area integrated quantity (with units of $\mathrm{kg} \mathrm{s}^{-1}$ ) as opposed to a true flux quantity (with units of $\mathrm{kg} \mathrm{s}^{-1} \mathrm{~m}^{-2}$ ). In this paper, we calculate the area integrated vertical mass flux as in RL11 and refer to the quantity as vertical mass flux (as in RL11) to maintain consistency.
}

Additionally, Smith and Montgomery (2012) and Montgomery and Smith (2012) argued that the tropical cyclogenesis model derived from the non-rotating, radiative convective equilibrium simulations presented in Raymond and Sessions (2007) is not strictly appropriate for off-equatorial tropical disturbances, such as the case of the pre-Typhoon Nuri disturbance. Finally, differences in data presented in RL11 and Raymond et al. (2011) lead to potential confusion on the dynamical mechanism of low-level spin-up. Specifically, Raymond et al. (2011) showed that the area-averaged low-level absolute vorticity increased from Nuri 1 to 2, contrary to their previous finding in RL11 that there was low-level spin-down. The area in which the average low-level absolute vorticity was calculated differs slightly between RL11 and Raymond et al. (2011), suggesting that part of the discrepancy may be due to the different averaging areas. Similarly, Raymond et al. (2011, their Fig. 9) showed that the average vorticity tendency was positive throughout the lower troposphere except at the surface (where it is $-0.001 \mathrm{ks}^{-1} \mathrm{dy}^{-1}$ or nearly zero). These results again differ from RL11 ${ }^{2}$. Given these apparent inconsistencies and the importance of the scientific issues summarized above, we believe there is a need for an independent assessment of the dynamics of Nuri's genesis. The objective of this study, then, is to answer the following questions:

(i) Was there spin-down on the system scale in the lower levels of the developing Typhoon Nuri disturbance between Nuri 1 and 2 as suggested by RL11?

(ii) Is the thermodynamic control hypothesis put forth by Raymond et al. (2011) a necessary condition for tropical cyclogenesis in the case of Typhoon Nuri?

(iii) How does the chosen area of integration for circulation budget calculations affect the results and the conclusions drawn from these analyses?

The outline of this paper is as follows: Sect. 2 discusses the data and methodology used in our analysis. Section 3 presents the results of our analysis for Nuri 1 and 2. Section 4 provides a discussion of our results and their implications on our understanding of Nuri's formation. Section 5 presents the conclusions.

\section{Data and methodology}

\subsection{Research flights and data collected}

The precursor easterly wave that contributed to the formation of Typhoon Nuri was first identified in operational forecast

\footnotetext{
${ }^{2}$ The differences in wave propagation speed used in RL11 $\left(-5 \mathrm{~m} \mathrm{~s}^{-1}\right)$ versus Raymond et al. (2011) $\left(-7 \mathrm{~m} \mathrm{~s}^{-1}\right)$ may have led to the small differences in magnitude of circulation (vorticity) tendency.
} 

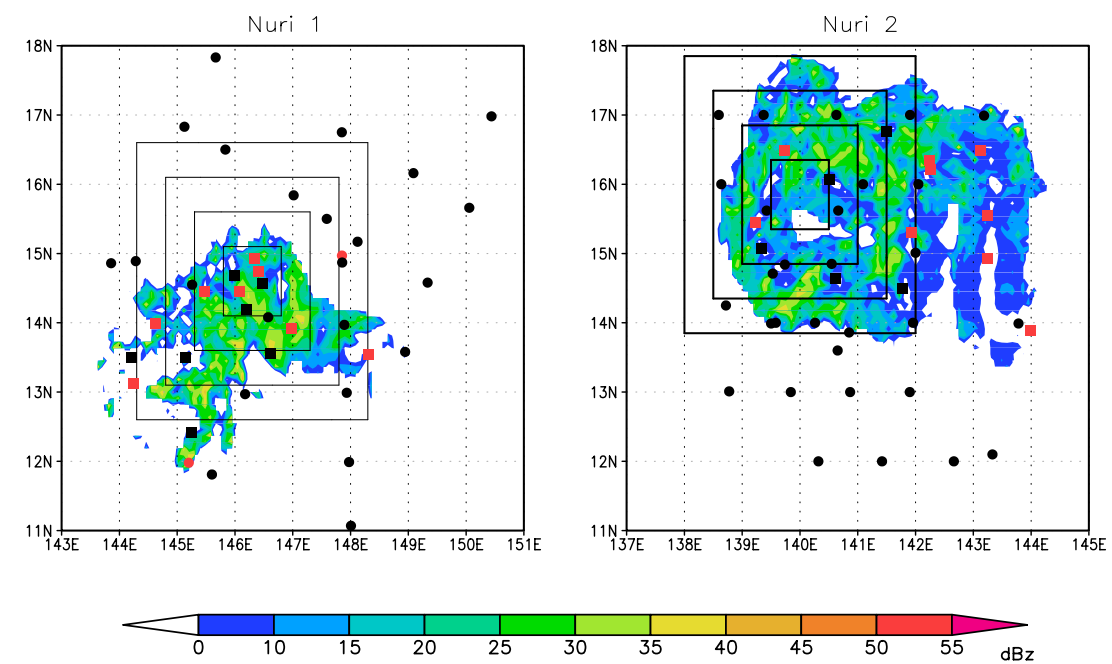

Figure 1. Electra-Doppler Radar reflectivity composite (in dBZ) at $3 \mathrm{~km}$ altitude for Nuri 1 (left) and Nuri 2 (right) superimposed with dropwindsonde locations. The dropwindsondes from the USAF C130 (filled circles) and NRL P3 (filled squares) have been translated to their 00:00 UTC positions using a (westward) phase speed of $-7 \mathrm{~m} \mathrm{~s}^{-1}$. The red circles and boxes indicate dropwindsondes with no retrieved wind data. The square boxes are at one-degree increments, increasing from one degree to four degrees, and represent the domain used in several of the forthcoming analyses. The boxes are centered on the sweet spot position at $1.5 \mathrm{~km}$ altitude within the pre-Nuri wave as derived from the $25 \mathrm{~km}$ SAMURAI analyses. The abscissa is longitude and the ordinate is latitude.

models on the morning of 12 August $2008^{3}$. The first TCS08 research flight was a joint United States Air Force (USAF) C130 and Naval Research Laboratory (NRL) P3 flight into the developing wave on 15-16 August 2008. The second research flight was flown on 16-17 August 2008 and was also a joint USAF C130, NRL P3 mission. The Joint Typhoon Warning Center (JTWC) upgraded the disturbance to a tropical depression (TD) at 12:00 UTC, 16 August 2008, between the first and second research flights. The third research flight included both aircraft and occurred between 17-18 August, after JTWC upgraded the disturbance to a tropical storm. The final research mission was a USAF C130-only mission on 18-19 August, when the disturbance was a typhoon. Following RL11 and consistent with the terminology defined in the Introduction, we refer to the state of Nuri observed during these four observation times as Nuri 1,2, 3 and 4, respectively. Since this paper is focused on the tropical cyclogenesis sequence and not on the subsequent intensification of the tropical cyclone, we will focus primarily on the data from the first two missions ${ }^{4}$.

The two types of data we use in this analysis are dropwindsonde observations from both the USAF C130 and NRL P3 and Electra-Doppler Radar (ELDORA) data from the NRL P3. The USAF C130 released the dropwindsondes from heights of up to $9.4 \mathrm{~km}$ while the NRL P3 typically operated

\footnotetext{
${ }^{3}$ Montgomery et al. (2010a) postulated that the wave originated on 7 August in the central Pacific.

${ }^{4}$ The exception is our Fig. 9, where for completeness and to compare our specific findings with RL11, we present the relative frequency distribution of vorticity for Nuri 1, 2 and 3.
}

at lower elevations (between 2.6 and $3.4 \mathrm{~km}$ ). The NRL P3 flew at higher elevations during the end of the first Nuri mission (approximately $7.3 \mathrm{~km}$ ) to collect more extensive dropwindsonde profiles. The ELDORA is an airborne dual-beam meteorological radar with an unambiguous range of $75 \mathrm{~km}$. Doppler radar data were collected in $150 \mathrm{~km}$ swaths centered on the P3 track (RL11). The radar data were edited using the quality control techniques described in Bell et al. (2013). Figure 1 illustrates the spatial coverage of the $\mathrm{C} 130$ dropwindsonde observations (filled circles), NRL P3 dropwindsondes (filled squares), and ELDORA coverage (as indicted by the $3 \mathrm{~km}$ altitude reflectivity return, dBZ) for Nuri 1 (left) and Nuri 2 (right). In all of the 3-D variational (3-D-VAR) analyses presented, these data are translated to their 00:00 UTC positions based on a zonal phase speed of $-7 \mathrm{~m} \mathrm{~s}^{-1}$. The thin boxes in Fig. 1 are centered on the sweet spot position at $1.5 \mathrm{~km}$ altitude out to four degrees length in one-degree increments. We choose the $1.5 \mathrm{~km}$ altitude sweet spot position for these calculations because the maximum amplitude of the pre-Nuri wave was in the lower troposphere (Montgomery et al., 2010a) ${ }^{5}$. The boxes are illustrative in nature and provide the reader with a sense of the data coverage at varying distances from the sweet spot. The forthcoming circulation tendency calculations are conducted on similar boxes at 0.2 degree increments from the sweet spot center. It is noted that

\footnotetext{
${ }^{5}$ The choice of $1.5 \mathrm{~km}$ altitude is consistent with the observed properties of easterly waves in the western North Pacific basin dating back to the 1970s, which found that the maximum amplitude of easterly waves was in the lower troposphere, at or below $850 \mathrm{hPa}$ (Reed and Recker, 1971; Chang, 1970).
} 
several of the dropwindsondes (indicated by red coloring) do not contain velocity data due to instrument errors.

To provide a foundation for assessing the changes in lowlevel spin-up between Nuri 1 and 2, we first carry out a circulation analysis using only dropwindsonde data. The coarse resolution and irregular spacing of the dropwindsonde data make identifying a sweet spot position challenging. To identify the cyclonic circulation center at each level, we follow the methodology of Davis and Ahijevych (2012). This method identifies the maximum-weighted average tangential wind within a three-degree radius circle at each location within the dropwindsonde domain. We perform this calculation at $5 \mathrm{hPa}$ intervals in the vertical and the horizontal location where this average tangential wind is maximized is designated as the cyclonic circulation center. The circulation centers at each level are used to examine the vertical tilt of the system. However, the $850 \mathrm{hPa}$ circulation center is chosen as the center position to calculate tangential wind at all levels for Nuri 1 and 2.

To compliment the dropwindsonde-only analysis, ELDORA and dropwindsonde data are analyzed together in a newly developed 3-D-VAR analysis scheme (Bell et al., 2012) referred to as SAMURAI (Spline Analysis at Mesoscale Using Radar and Aircraft Instrumentation). The SAMURAI analysis uses a Bayesian approach to determine the maximum likelihood estimate of the thermodynamic and kinematic data from the observations (Bell et al., 2012). SAMURAI is similar to other variational schemes that minimize the difference between observations and the resulting analysis, but unlike numerical weather prediction data assimilation it enforces no balance or physical constraints other than mass continuity. The analysis scheme uses a series of finite elements in the form of cubic B-splines to represent the 3-D atmospheric state. The spline analysis has the advantage of near-analytic derivatives for vorticity and divergence, and precise interpolation to the observation space for irregularly spaced data such as dropwindsonde and airborne radar data. SAMURAI bears some similarity to the 3-D-VAR software used in RL11 (López-Carrillo and Raymond, 2011), but has a distinctly different numerical implementation of the variational cost function. No fundamental differences between the wind fields presented here and those presented in R11 were apparent, suggesting that both analysis techniques capture the salient features of Nuri, but differ in the details.

Several SAMURAI analyses were created for this study. For each research flight, a synoptic-scale analysis, with horizontal grid spacing of $25 \mathrm{~km}$, and a mesoscale analysis, with horizontal grid spacing of $10 \mathrm{~km}$, were created. A Gaussian low-pass filter was applied to the analyses to spread information from the observations and reduce noise in the wind fields. The horizontal filter lengths were 125 and $50 \mathrm{~km}$ for the synoptic-scale and mesoscale analyses, respectively. All analyses have $0.5 \mathrm{~km}$ vertical grid spacing from the surface to an upper height of $16.5 \mathrm{~km}$, with a $1 \mathrm{~km}$ vertical filter length. In the forthcoming analyses, we only present data up to $12 \mathrm{~km}$ in the vertical due to the high-elevation sampling limitations of the ELDORA. The main purpose of the $25 \mathrm{~km}$ analyses is to identify the sweet spot position during Nuri 1 and 2 as it is hypothesized to be the region for upscale vorticity growth and organization (DMW09, Montgomery et al., 2010b). The $10 \mathrm{~km}$ analyses are used primarily to construct the kinematic and dynamic variables for the vorticity analysis.

\subsection{Vorticity and circulation dynamics}

The vorticity dynamics are diagnosed using the $10 \mathrm{~km}$ SAMURAI analyses following RL11. Following Haynes and McIntyre (1987) and RL11, the vertical vorticity equation may be rewritten into the flux form as follows:

$\frac{\partial \eta}{\partial t}=-\nabla_{\mathrm{h}} \cdot \boldsymbol{Z}+\hat{\boldsymbol{k}} \cdot \nabla_{\mathrm{h}} \theta \times \nabla_{\mathrm{h}} \Pi$,

where $\eta$ is the absolute vertical vorticity, the subscript $h$ represents the horizontal vector quantity, and $\theta$ is potential temperature. The Exner function is defined as follows: $\Pi=\left(\frac{p}{p_{0}}\right)^{R_{\mathrm{d}}} / c_{\mathrm{p}}$, where $p$ is the pressure, $p_{0}$ is the pressure at a reference level (typically $1000 \mathrm{hPa}$ ), $R_{\mathrm{d}}$ is the gas constant for dry air, and $c_{\mathrm{p}}$ is the heat capacity of dry air at constant pressure. The vector vorticity flux, $\boldsymbol{Z}$, is defined as follows:

$\boldsymbol{Z}=Z_{1}+Z_{2}+Z_{3}=\eta_{z} \boldsymbol{v}_{\boldsymbol{h}}-\boldsymbol{\eta}_{\mathrm{h}} v_{z}+\hat{\boldsymbol{k}} \times \boldsymbol{F}$,

where the z-subscript represents the vertical component of the vector and the vector $\boldsymbol{F}$ represents horizontal frictional and other sub-grid-scale forces. If we assume that $\boldsymbol{F}$ can be approximated by friction only, it can be parameterized in a simple and plausible way (following RL11) as follows:

$\boldsymbol{F}=\frac{\boldsymbol{\tau}}{\rho} \exp \left(-z / z_{\mathrm{s}}\right) / z_{\mathrm{s}}$,

where $\rho$ is air density, $z$ is height, $z_{\mathrm{s}}$ is a characteristic boundary layer height $(1.25 \mathrm{~km})$ and the surface stress $\tau$ is defined as follows:

$\boldsymbol{\tau}=-\rho_{\mathrm{BL}} C_{\mathrm{D}}\left|\boldsymbol{U}_{\mathrm{BL}}\right| \boldsymbol{U}_{\mathrm{BL}}$,

where the subscript $\mathrm{BL}$ represents conditions in the boundary layer, $\boldsymbol{U}$ is the horizontal wind and $C_{\mathrm{D}}$ is the surface drag coefficient, defined as follows:

$C_{\mathrm{D}}=\left(1+0.028\left|\boldsymbol{U}_{\mathrm{BL}}\right|\right) \times 10^{-3}$

Now, integrating Eq. (1) over a horizontal surface, and neglecting the small contribution from the baroclinic vorticity generation term, results in the circulation tendency equation:

$\frac{d \Gamma_{\mathrm{a}}}{d t}=\int_{A} \nabla \cdot \boldsymbol{Z} d A$, 
where $\Gamma_{\mathrm{a}}$ is the absolute circulation and $A$ is the area over which the integration is performed (in this work, a square box). By applying Gauss's divergence theorem, the circulation tendency can also be calculated using the line integral form:

$\frac{d \Gamma_{\mathrm{a}}}{d t}=-\oint v_{n} \eta_{z} d l+\oint \eta_{n} v_{z} d l+\oint F_{t} d l$

where $d l$ is the positive line element along the perimeter of the integration area, the subscript $n$ denotes the outward normal component of the variable along the circuit, and the subscript $t$ denotes the tangential component of the variable in the sense of the circuit. The integration is taken in a counterclockwise sense in accordance with the right-hand rule. Equation (7) shows that changes to the absolute circulation occur through (i) convergence of absolute vorticity (first term); (ii) vortex tilting-like term (second term); and iii) the frictional spin-down tendency (third term). RL11 argue that the spin-up of a vortex in the lower levels can occur only if the spin-up from convergence of absolute vorticity exceeds the frictional spin-down tendency ${ }^{6}$. Davis and Galarneau (2011) show that the convergence term can be broken down into "mean" and "eddy" contributions along the circuit:

$-\oint v_{n} \eta_{z} d l=-\bar{\eta} \tilde{\delta} A-\oint \eta^{\prime} \boldsymbol{v}^{\prime} \cdot \hat{\boldsymbol{n}} d l$

where $\delta$ is the horizontal divergence, the overbars represent the mean over the perimeter of the circuit, the primes indicate perturbations from this mean, the tildes indicate an areal average over the circuit, and $\boldsymbol{v}$ is shorthand for the horizontal velocity vector. The first term on the RHS of Eq. (8) represents vortex-tube stretching averaged over the area contained within the circuit, while the second term in this equation represents the change in the absolute circulation owed to horizontal eddy fluxes of vertical vorticity into and out of the integration area.

\footnotetext{
${ }^{6}$ Several previous studies (Willoughby, 1995; Schubert and Alworth, 1987; Marín et al., 2009) share a similar view with RL11 and Raymond et al. (2011) in that they suggest that friction within the boundary layer acts solely as a detriment to spin-up on the systemscale circulation. We do not dispute the strict statement that spinup in the boundary layer only occurs when convergence of absolute vorticity is larger than the tendency to spin down the flow due to friction. It is not true, however, that the dynamics of the frictional boundary layer act solely as a detriment to spin-up. Smith et al. (2009), Sanger et al. (2013), and Montgomery and Smith (2014) illustrate that within the inner-core region of a developing tropical cyclone vortex, the frictional inflow acquires relatively significant values compared to the tangential component. These latter studies demonstrate a dynamical role of the boundary layer, wherein air parcels may reach small radii quickly (minimizing the loss of absolute angular momentum (defined in Sect. 3) during spiral circuits) and therefore acquiring large $v$ that may be larger than the $v$ in the interior flow (and the gradient value thereof) above the boundary layer.
}

One of the objectives of this work is to quantify how the circulation dynamics vary within the wave pouch at different distances from the sweet spot. With this objective in mind, the SAMURAI analyses are used to calculate terms from the circulation tendency equation (Eq. 7) and other variable properties in 0.2 degree length boxes centered on the $1.5 \mathrm{~km}$ altitude sweet spot position. The advantages of this analysis methodology are (i) it is a systematic way to analyze the kinematics and dynamics at varying distances from the sweet spot position; (ii) it allows for a straightforward comparison of the various spin-up contributions between research flights; (iii) the methodology is optimally suited to observe and quantify the development that ensues around the sweet spot, the so-called "attractor point" predicted by the new cyclogenesis model (Montgomery et al., 2012); and (iv) anchoring our analysis to the sweet spot of the parent disturbance provides useful information on the vertical alignment of the vortex and depth of the wave pouch. The second and third points are especially important as there is considerable variability in the areal coverage of the aircraft data during the Nuri missions (Fig. 1).

The main limitation of this type of analysis is that the observed data are not distributed uniformly over the defined boxes. This results in data being unavailable in small regions in some of the outer boxes. To address this issue, in all of the calculations performed in this paper, regions outside of the observational boundaries are set to missing values. Since these issues arise either at the outer edge, or outside of the wave pouch, we believe that the impact on the analysis presented herein is minimal. Non-uniform data distribution is unfortunately a limitation in any observational study and we feel that the advantages gained in this type of analysis far outweigh the disadvantages.

\section{Results}

\subsection{Dropwindsonde-only analysis}

Dropwindsonde analysis from the first research flight into the pre-Nuri (Nuri 1) disturbance is presented in Fig. 2. This analysis depicts wind barbs in the co-moving frame at standard pressure levels up to $500 \mathrm{hPa}$. The black circles indicate the position of the $850 \mathrm{hPa}$ circulation center and the blue triangles indicate the position of the circulation center at the indicated level. The low-level (at and below $850 \mathrm{hPa}$ ) wind fields indicate a closed, cyclonic circulation in the comoving frame (Fig. 2, top). The $850 \mathrm{hPa}$ circulation center is located near $14.7^{\circ} \mathrm{N}, 146.8^{\circ} \mathrm{E}$ and is nearly vertically aligned down to the $925 \mathrm{hPa}$ level. At 700 and $500 \mathrm{hPa}$, there are cyclonic circulation centers that tilt eastward with height. At $500 \mathrm{hPa}$, the cyclonic circulation center is located near $14.7^{\circ} \mathrm{N}, 149.4^{\circ} \mathrm{E}$, a 2.6 degree eastward tilt with increasing height. 

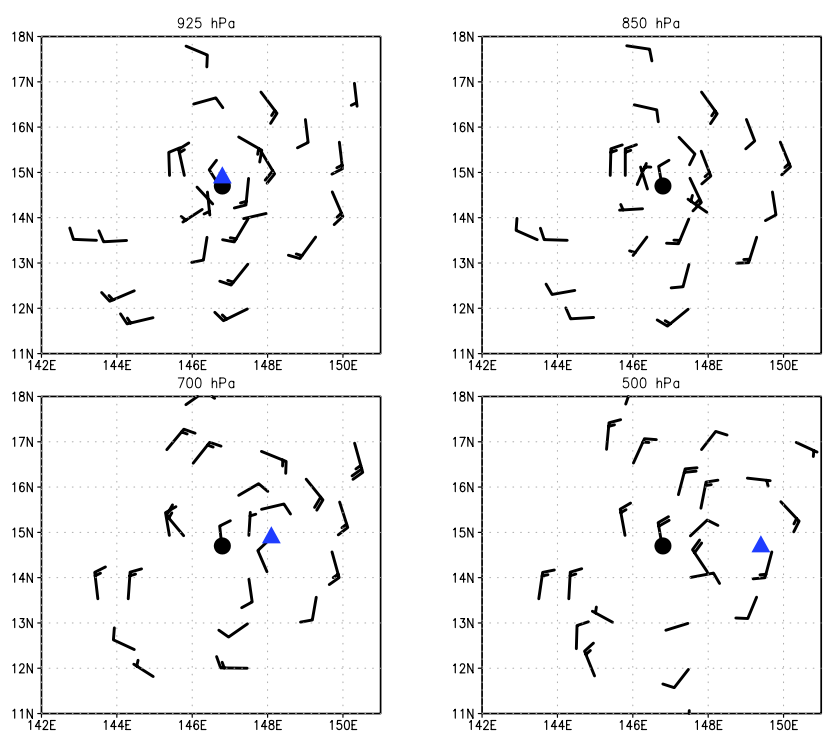

Figure 2. Four-panel plot of wind barbs from the dropwindsonde observations in the co-moving frame for Nuri 1. Levels plotted are $925 \mathrm{hPa}$ (a), $850 \mathrm{hPa}$ (b), $700 \mathrm{hPa}$ (c), and $500 \mathrm{hPa}$ (d). In this figure and Fig. 3, the winds are in standard convention for wind barbs (kt - knot), rather than the SI units used in the remained of the paper. A full wind barb denoted $10 \mathrm{kt}$ and each half barb is $5 \mathrm{kt}$. The filled circle represents the $850 \mathrm{hPa}$ dropwindsonde-derived cyclonic circulation center and the filled triangles are the circulation centers at the indicated pressure level. The abscissa is longitude and the ordinate is latitude.
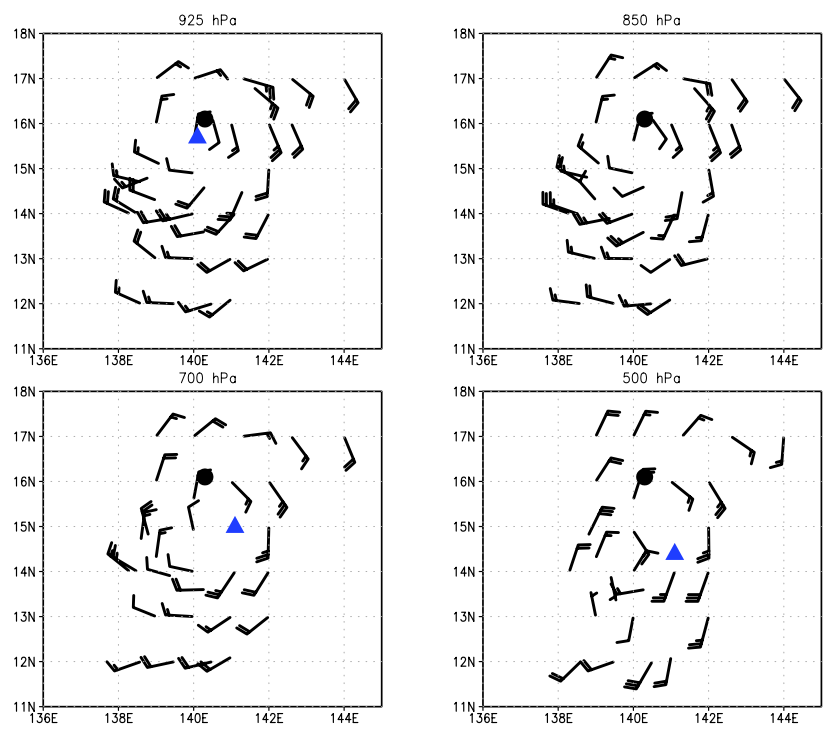

Figure 3. Dropwindsonde observations from the Nuri 2 flight (as in Fig. 2).

Figure 3 is similar to Fig. 2, but for Nuri 2. The $850 \mathrm{hPa}$ cyclonic circulation center is located near $16.1^{\circ} \mathrm{N}, 140.3^{\circ} \mathrm{E}$ and remains nearly vertically aligned down to $925 \mathrm{hPa}$. The circulation center has become more longitudinally aligned with the low-level circulation center at 700 and $500 \mathrm{hPa}$, but there is now a distinct tilt to the southeast with height. At $500 \mathrm{hPa}$, the cyclonic circulation center is located near $14.1^{\circ} \mathrm{N}, 141.1^{\circ} \mathrm{E}$, a 2.2 degree tilt with height. From a qualitative point of view, since the average wind speed appears to have increased at all levels from Nuri 1 to 2, there does not appear to be low-level spin-down between these two missions.

Figure 4 is a 4-panel plot of tangential wind profiles derived from the dropwindsonde data at one-degree radius intervals for the first two Nuri missions. For each dropwindsonde, the tangential wind is calculated at each level in the vertical and then binned in $5 \mathrm{hPa}$ intervals. An average tangential wind is then calculated in each bin. The binning is accomplished to smooth high-resolution variations in these data and as an attempt to limit the impact of missing data when direct comparisons are made between dropwindsonde observations at particular levels. An azimuthal average is then performed within one-degree radius annuli from the $850 \mathrm{hPa}$ dropwindsonde-derived circulation center.

The maximum tangential wind for Nuri 1 occurs within the $2-3$ degree radius annulus, near $850 \mathrm{hPa}$. The inner two annuli indicate areas of positive tangential wind up to approximately $600 \mathrm{hPa}$. Above this level, there are both positive and negative values of tangential wind, likely due to the tilt of the vortex with height discussed above. The largest increase in tangential wind between Nuri 1 and 2 occurs in the 1-2 degree radius annulus at $600 \mathrm{hPa}$. For Nuri 2, the radius of maximum tangential wind is less well defined, as all radii beyond one degree indicate similar wind speeds. The $1 \mathrm{de}-$ gree and 2-3 degree annuli show slight decreases in tangential wind speed from Nuri 1 to 2 between $800-700 \mathrm{hPa}$. The distribution of dropwindsonde observations for Nuri 2 in the outermost radius (Fig. 4d) displays a strong sampling bias to the south of the sweet spot position. While these data are shown for completeness, it is difficult to draw robust conclusions with respect to the system-scale circulation in the outer-most radius of Nuri 2. The most important feature evident in these profiles is that there is an increase in observed tangential wind speed at and below $800 \mathrm{hPa}$ from Nuri 1 to 2 at all radii. These observations suggest that spin-up within and just above the boundary layer is occurring between these two missions.

\subsection{SAMURAI analysis}

To test the robustness of the finding that low-level spin-up is occurring between the first two missions, the SAMURAI analyses are examined next. Figure 5 shows a two-panel plot of the Okubo-Weiss (OW) parameter, streamlines in the comoving frame, and isotachs at 1.5 and $5 \mathrm{~km}$ altitude from the Nuri 1 mission $25 \mathrm{~km}$ analysis. Okubo-Weiss is defined as $\mathrm{OW}=\zeta^{2}-S_{1}^{2}-S_{2}^{2}=\left(V_{\mathrm{x}}-U_{\mathrm{y}}\right)^{2}-\left(U_{\mathrm{x}}-V_{\mathrm{y}}\right)^{2}-\left(V_{\mathrm{x}}+U_{\mathrm{y}}\right)^{2}$, where $\zeta$ is relative vorticity, $S_{1}$ and $S_{2}$ are strain deformation, $(U, V)$ are zonal and meridional wind, and the subscripts 

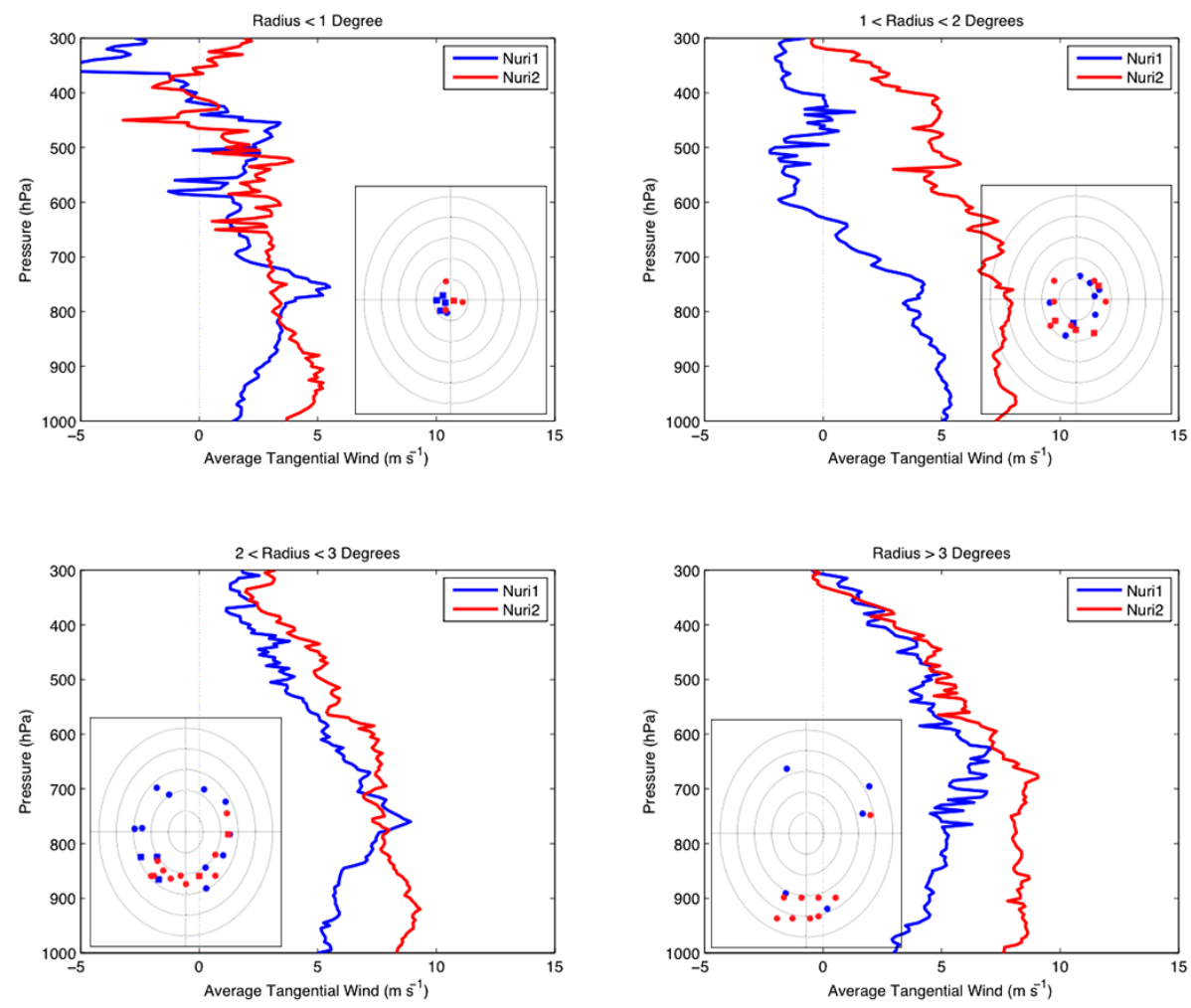

Figure 4. Four-panel plot of dropwindsonde-derived azimuthally averaged tangential wind profiles for the first two research flights into the pre-Nuri disturbance. The blue curve is Nuri 1 and the red curve is Nuri 2. The average profiles are calculated in one-degree radius annuli from the dropwindsonde-derived $850 \mathrm{hPa}$ circulation center (filled circles in Figs. 2 and 3) for each research flight. The insets indicate dropwindsonde positions relative to the low-level circulation center for each annulus. Each circle is one degree radius. The blue circles are the Nuri 1 USAF C130 dropwindsonde positions and the blue squares are the Nuri 1 NRL P3 dropwindsonde positions. The circles and squares follow the same convention for the Nuri 2 dropwindsonde positions.

$(x, y)$ indicate partial derivatives in the zonal and meridional directions, respectively. Significantly positive regions of OW indicate areas that will tend to be immune from horizontal shearing deformation, while negative regions indicate areas subject to shearing deformation (McWilliams, 1984; Weiss, 1991). The positions of the wave trough (locus of points with $v=0$ ) and critical surface (locus of points with zero relative zonal wind) at $1.5 \mathrm{~km}^{7}$ are indicated by the thick black and the purple lines, respectively. For Nuri 1, the $1.5 \mathrm{~km}$ sweet spot position is located near $14.6^{\circ} \mathrm{N}, 146.3^{\circ} \mathrm{E}$. The center of the $5 \mathrm{~km}$ circulation (as determined by the comoving streamlines) is near $12.9^{\circ} \mathrm{N}, 147.9^{\circ} \mathrm{E}$. This indicates a larger tilt to the southeast with height than observed in the dropwindsonde-only data. Although the exact positions of the circulation centers are slightly different from those in RL11, the overall flow pattern is fairly consistent (cf. RL11, p. 155). At $1.5 \mathrm{~km}$, the OW maximum is centered near the sweet spot position while there are several OW maxima at

\footnotetext{
${ }^{7}$ While the dropwindsonde data are displayed on pressure levels in the vertical, the SAMURAI data are displayed in height. The $1.5 \mathrm{~km}$ height corresponds to approximately the $850 \mathrm{hPa}$ level and the $5 \mathrm{~km}$ height corresponds to approximately the $550 \mathrm{hPa}$ level.
}

$5 \mathrm{~km}$. A similar analysis for Nuri 2 (Fig. 6) indicates a $1.5 \mathrm{~km}$ sweet spot position at $15.9^{\circ} \mathrm{N}, 140^{\circ} \mathrm{E}$. The $5 \mathrm{~km}$ cyclonic circulation has now become longitudinally aligned with the low-level circulation and is located near $14.1^{\circ} \mathrm{N}, 140.3^{\circ} \mathrm{E}$. The OW field has become more organized and concentrated near the cyclonic circulation center at both levels.

To examine the observed structure of vertical vorticity and horizontal wind within the wave pouch region, Figs. 7 and 8 show two-panel plots of absolute vorticity and wind vectors (co-moving) from the $10 \mathrm{~km}$ SAMURAI analyses at 1.5 and $5 \mathrm{~km}$ for Nuri 1 and 2. For Nuri 1, the $1.5 \mathrm{~km}$ mesoscale cyclonic circulation center appears north of the $25 \mathrm{~km}$-analysis sweet spot position. This mesoscale circulation center is associated with a positive vorticity maximum on a similar horizontal scale. The presence of mesoscale circulation centers that are not co-located with the sweet spot is not uncommon, as multiple studies (Wang et al., 2009; Lussier III, 2010) have shown the presence of short-lived, smaller-scale circulations within the larger wave pouch. The $5 \mathrm{~km}$ cyclonic circulation center appears to be southeast of the low-level circulation center. The mesoscale absolute vorticity maxima at $5 \mathrm{~km}$ are aligned well with the maxima identified in the $25 \mathrm{~km}$ 

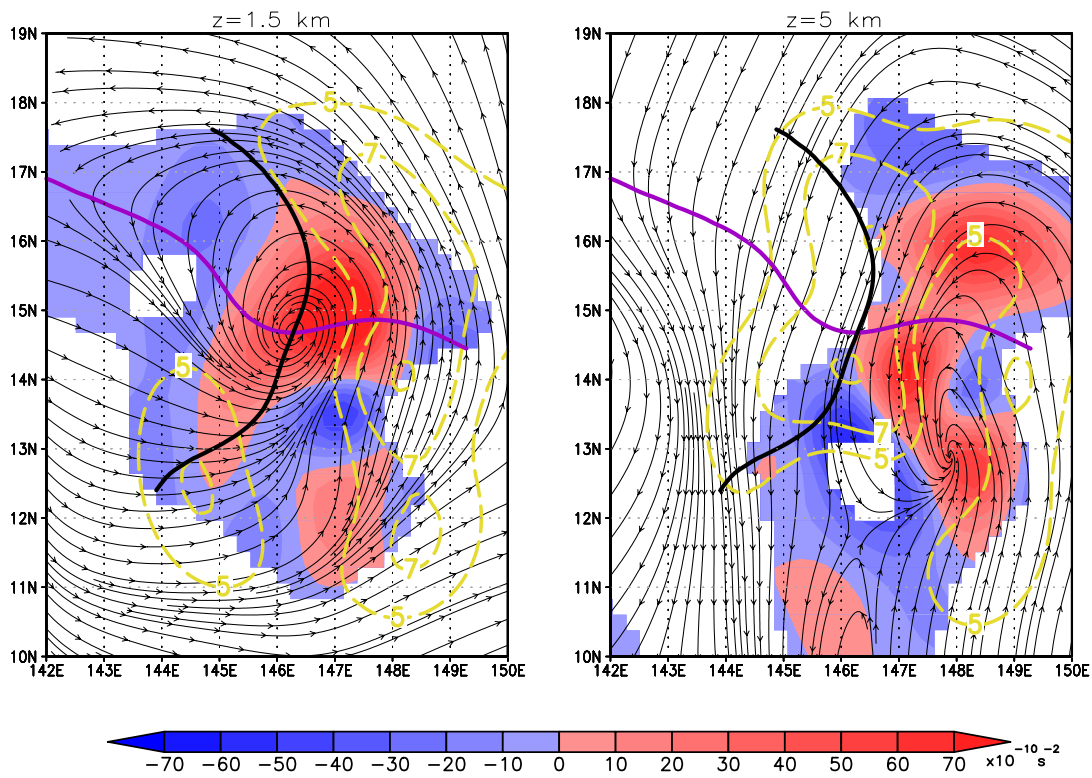

Figure 5. Two-panel plot of Okubo-Weiss (OW, shaded) and streamlines (co-moving) for Nuri 1 at $1.5 \mathrm{~km}$ (left) and $5 \mathrm{~km}$ (right) height from the $25 \mathrm{~km}$ SAMURAI analysis. The thick purple line indicates the position of the $1.5 \mathrm{~km}$ critical latitude and the thick black line indicates the position of the $1.5 \mathrm{~km}$ wave trough. The dashed yellow lines are isotachs contoured at $2 \mathrm{~m} \mathrm{~s}^{-1}$ intervals. The OW is only shaded in regions with cyclonic relative vorticity. The abscissa is longitude and the ordinate is latitude.
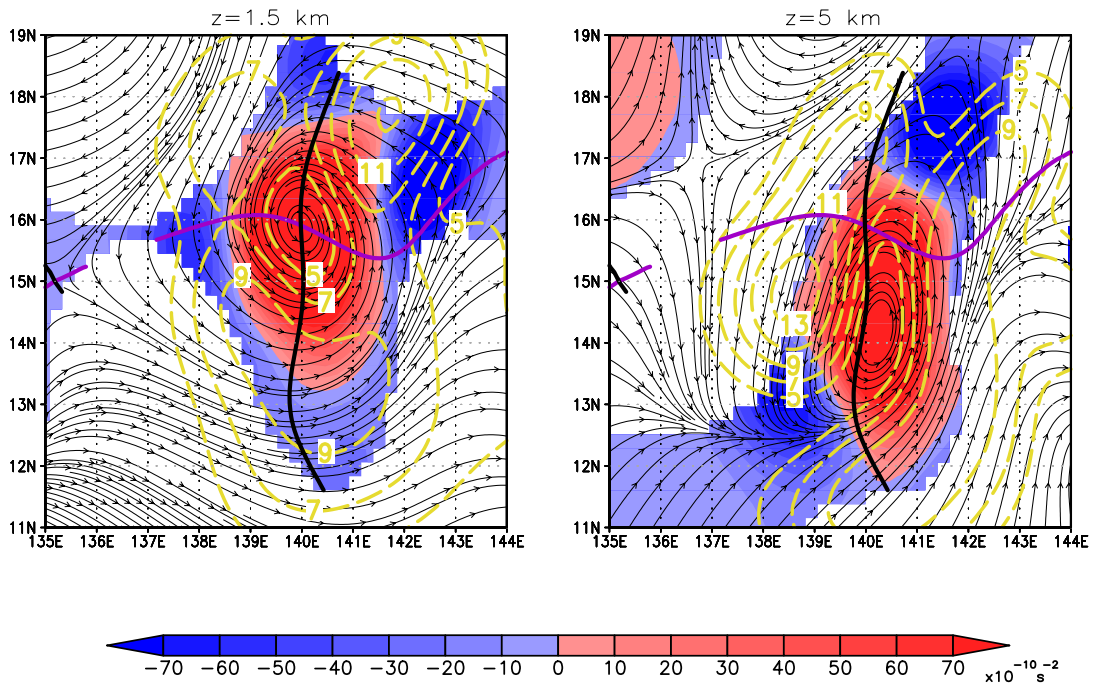

Figure 6. Two-panel plot of Okubo-Weiss (OW, shaded) and streamlines (co-moving) for Nuri 2 at $1.5 \mathrm{~km}$ (left) and $5 \mathrm{~km}$ (right) height from the $25 \mathrm{~km}$ SAMURAI analysis. The thick purple line indicates the position of the $1.5 \mathrm{~km}$ critical latitude and the thick black line indicates the position of the $1.5 \mathrm{~km}$ wave trough. The dashed yellow lines are isotachs contoured at $2 \mathrm{~ms}^{-1}$ intervals. The OW is only shaded in regions with cyclonic relative vorticity. The abscissa is longitude and the ordinate is latitude.

analysis. The overall wind field and vorticity pattern are consistent with the analysis from RL11. However, the magnitude of the absolute vorticity is generally weaker in our analyses. The Nuri 2 wind field exhibits a more coherent circulation than observed in the Nuri 1 analysis. In particular, the $1.5 \mathrm{~km}$ vorticity maxima have become more consolidated around the sweet spot position. There are localized low-level absolute vorticity maxima located near $14.3^{\circ} \mathrm{N}, 140.3^{\circ} \mathrm{E}$ and $16.8^{\circ} \mathrm{N}$, $140.3^{\circ}$ E. At $5 \mathrm{~km}$ the circulation has become more vertically aligned with height in the longitudinal direction and multiple vorticity maxima are observed within the wave pouch.

To help quantify the statistical evolution of cyclonic relative vorticity that takes place during the research flights, Fig. 9 shows a 4-panel plot of the relative frequency of 

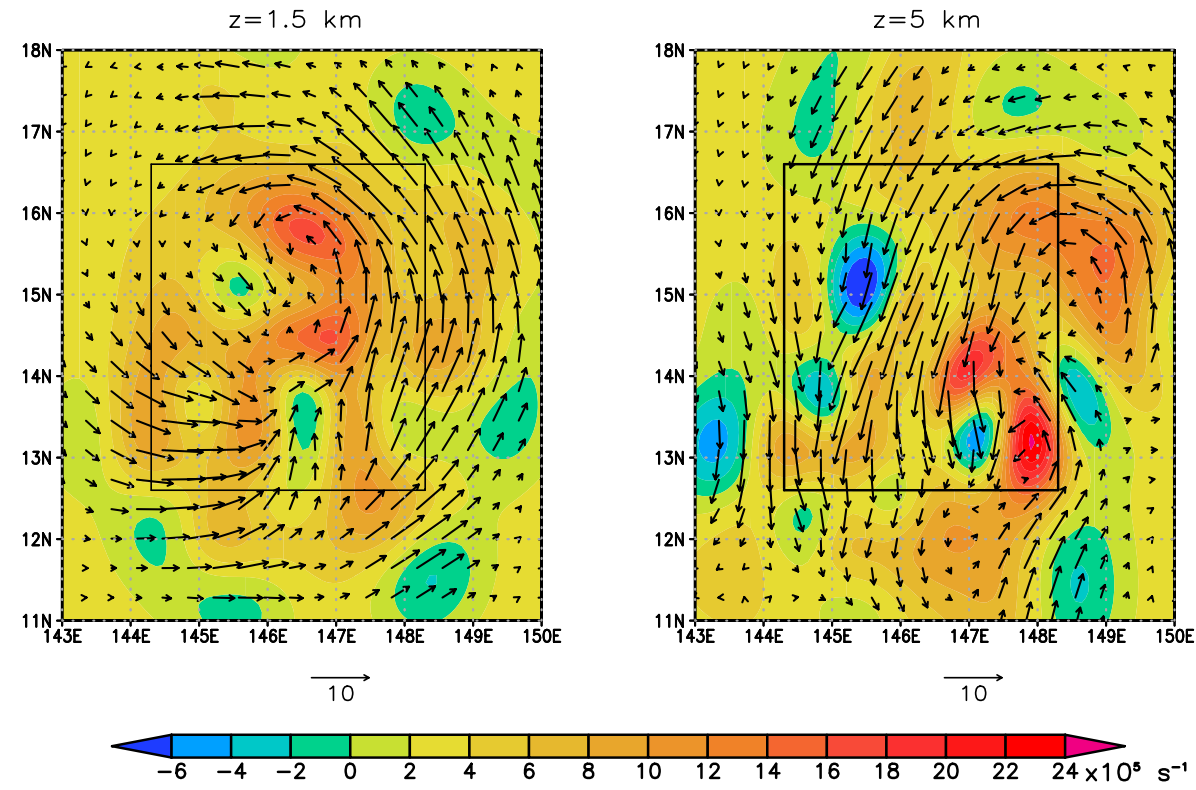

Figure 7. Two-panel plot of absolute vorticity (shaded) and wind vectors (co-moving) for Nuri 1 at $1.5 \mathrm{~km}$ (left) and $5 \mathrm{~km}$ (right) height from the $10 \mathrm{~km}$ SAMURAI analyses. The black box is the four-degree length box centered on the $25 \mathrm{~km}$ analysis $1.5 \mathrm{~km}$ altitude sweet spot position and is for reference only. The abscissa is longitude and the ordinate is latitude.

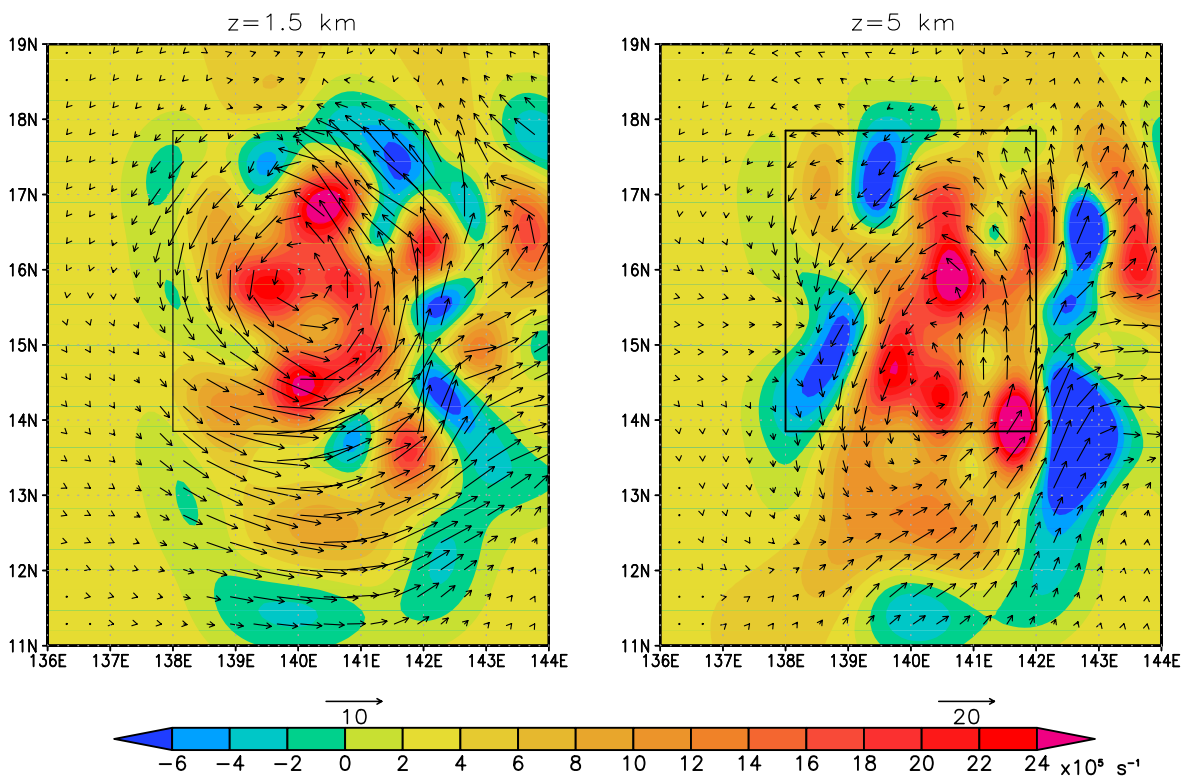

Figure 8. Two-panel plot of absolute vorticity (shaded) and wind vectors (co-moving) for Nuri 2 at $1.5 \mathrm{~km}$ (left) and $5 \mathrm{~km}$ (right) height from the $10 \mathrm{~km}$ SAMURAI analyses. The black box is the four-degree length box centered on the $25 \mathrm{~km}$ analysis $1.5 \mathrm{~km}$ altitude sweet spot position and is for reference only. The abscissa is longitude and the ordinate is latitude.

absolute vorticity for the first three Nuri missions derived from the $10 \mathrm{~km}$ SAMURAI analyses. Frequency distributions of absolute vorticity within $0.5,1,1.5$ and 2 degrees radius of the $1.5 \mathrm{~km}$ sweet spot position are plotted. For the case of Nuri 3, the sampling area is biased to the northeast of the circulation center (RL11, Fig. 7), and the observa- tional coverage within the two-degree radius circles is less than in Nuri 1 and 2. This, combined with the bin sizes used to construct the histogram, leads to some of the noisiness seen in the Nuri 3 traces. The shape of the distributions in the largest radius agrees fairly well with the distributions in Fig. 10 of RL11. The most noticeable exception is that the 

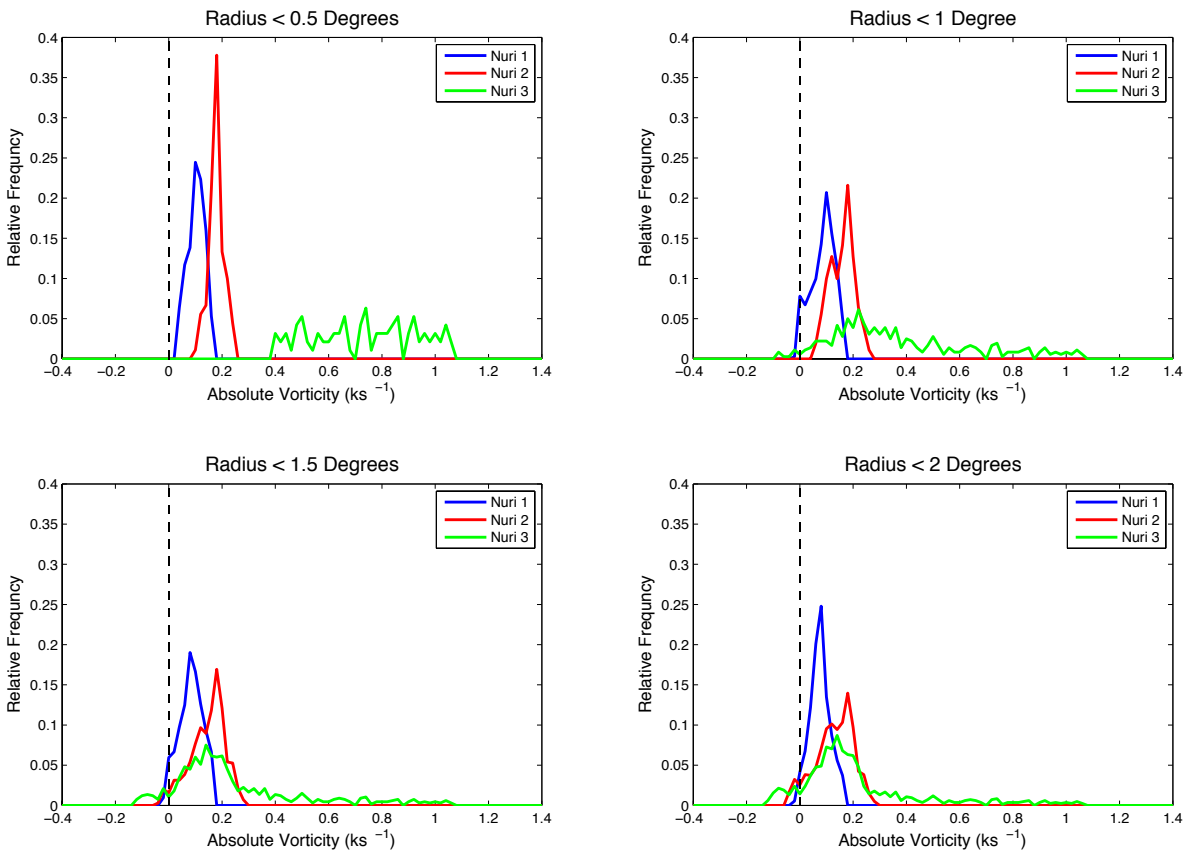

Figure 9. Four-panel plot of relative frequency of absolute vorticity for Nuri 1 (blue), Nuri 2 (red), and Nuri 3 (green) derived from the $10 \mathrm{~km}$ SAMURAI analyses. The relative frequency distributions are calculated in 0.5 degree radius intervals from the $1.5 \mathrm{~km}$ altitude sweet spot position out to two degrees. The dashed black line represents the zero absolute vorticity value.
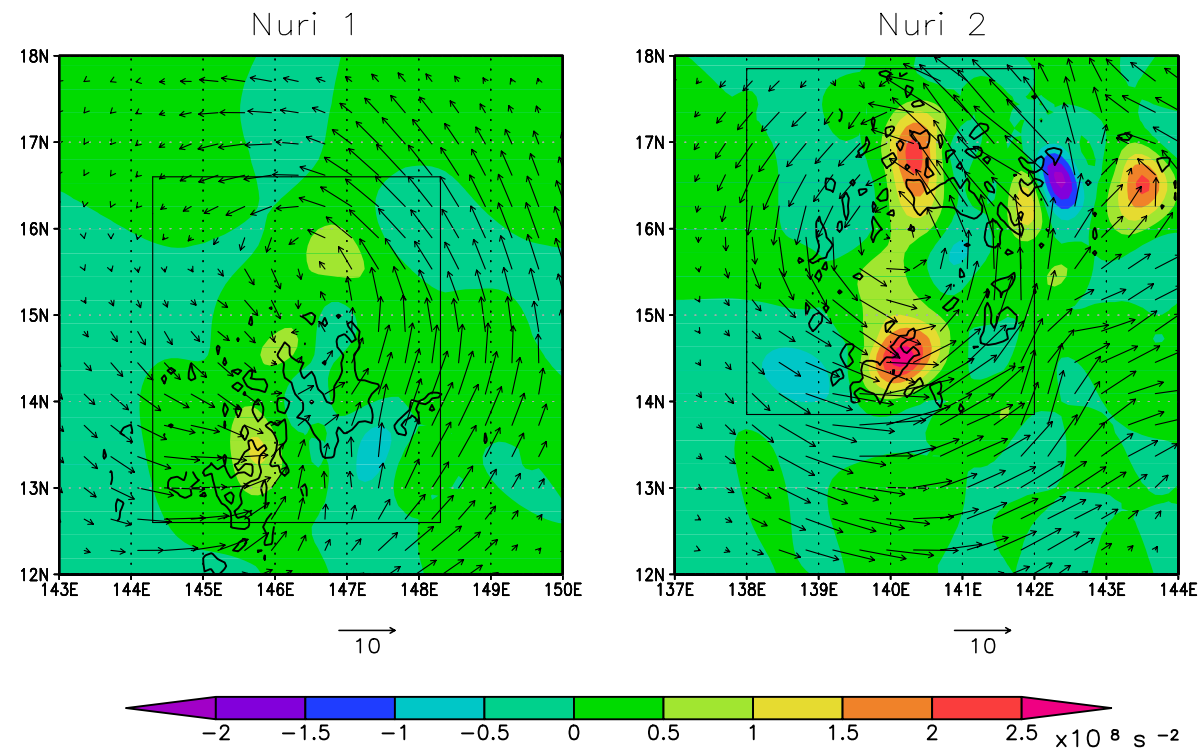

Figure 10. Two-panel plot of low-level stretching tendency (shaded) for Nuri 1 (left) and Nuri 2 (right). The maximum stretching tendency between the surface and $1 \mathrm{~km}$ is displayed. The arrows are wind vectors in the co-moving frame at $1.5 \mathrm{~km}$ altitude and the thick black lines are the $25 \mathrm{dBZ}$ contour from the ELDORA data at $5 \mathrm{~km}$. The black box is the four-degree length box centered on the $1.5 \mathrm{~km}$ sweet spot position and is for reference only. The abscissa is longitude and the ordinate is latitude.

Nuri 3 distribution does not have a large negative area in our plot. This is likely attributed to plotting the data by radius, rather than by the entire sampled area. Many of the negative values in RL11's distribution appear to be located beyond the two-degree radius. This could lead to a different interpretation of the results and illustrates one of the advantages in averaging over discrete radial intervals. In Fig. 9, the distribution maximum for Nuri 1 remains in essentially the same location at each radius; however, the spread in the distribution decreases at the 0.5 degree radius. For Nuri 1, this 

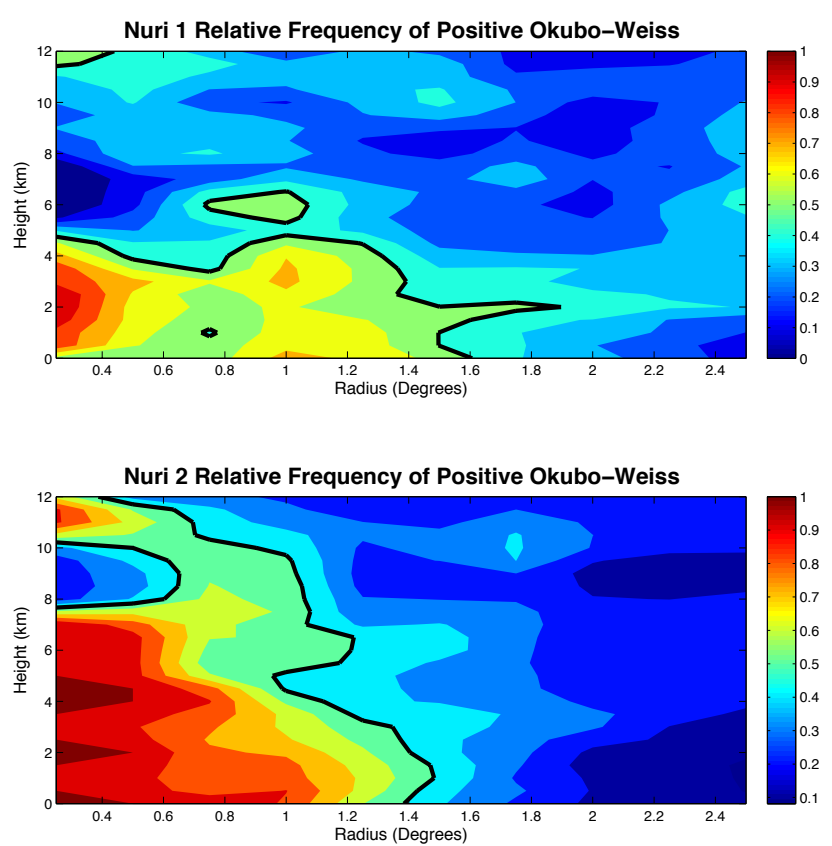

Figure 11. Two-panel vertical cross section of the frequency of positive $\mathrm{OW}$ values. The abscissa is radial distance from the $1.5 \mathrm{~km}$ derived sweet spot position in degrees and the ordinate is height $(\mathrm{km})$. The thick black line represents the 0.5 contour (i.e., the location where the frequency of occurrence of positive OW is equal to the frequency of occurrence of negative $\mathrm{OW}$ ).

suggests a somewhat uniform vorticity distribution throughout the wave pouch with no strong maxima. The spread of the Nuri 2 distribution also decreases with decreasing radius. This suggests that, over time, low-level cyclonic vorticity is concentrated near the sweet spot position. By the Nuri 3 mission, the vortex is well organized, and this is represented by relatively large values of low-level cyclonic absolute vorticity in the inner-most radius.

Figure 10 shows the low-level stretching tendency for Nuri 1 and 2 overlaid with wind vectors in the co-moving frame and the $5 \mathrm{~km} 25 \mathrm{dBZ}$ contour. The stretching tendency is calculated following RL11, where the divergence of the advective flux of vorticity is:

$-\nabla \cdot Z_{1}=-\boldsymbol{v}_{\boldsymbol{h}} \cdot \nabla_{\mathrm{h}} \eta_{z}-\eta_{z} \delta$

The first term on the LHS of Eq. (9) is the advection of absolute vertical vorticity in the horizontal, a process that merely redistributes the vorticity in the horizontal plane. The second term is typically referred to as the stretching term. Vertical stretching (compression) of the column can lead to a localized increase (decrease) in the vorticity magnitude. Areas of maximum positive stretching tendency are observed within the wave pouch near $13.4^{\circ} \mathrm{N}, 145.8^{\circ} \mathrm{E}$ in Nuri 1 and $14.5^{\circ} \mathrm{N}, 140.1^{\circ} \mathrm{E}$ and $16.8^{\circ} \mathrm{N}, 140.3^{\circ} \mathrm{E}$ during Nuri 2, and these maxima are associated with cumulus convection as evidenced by their approximate co-location with high radar re-

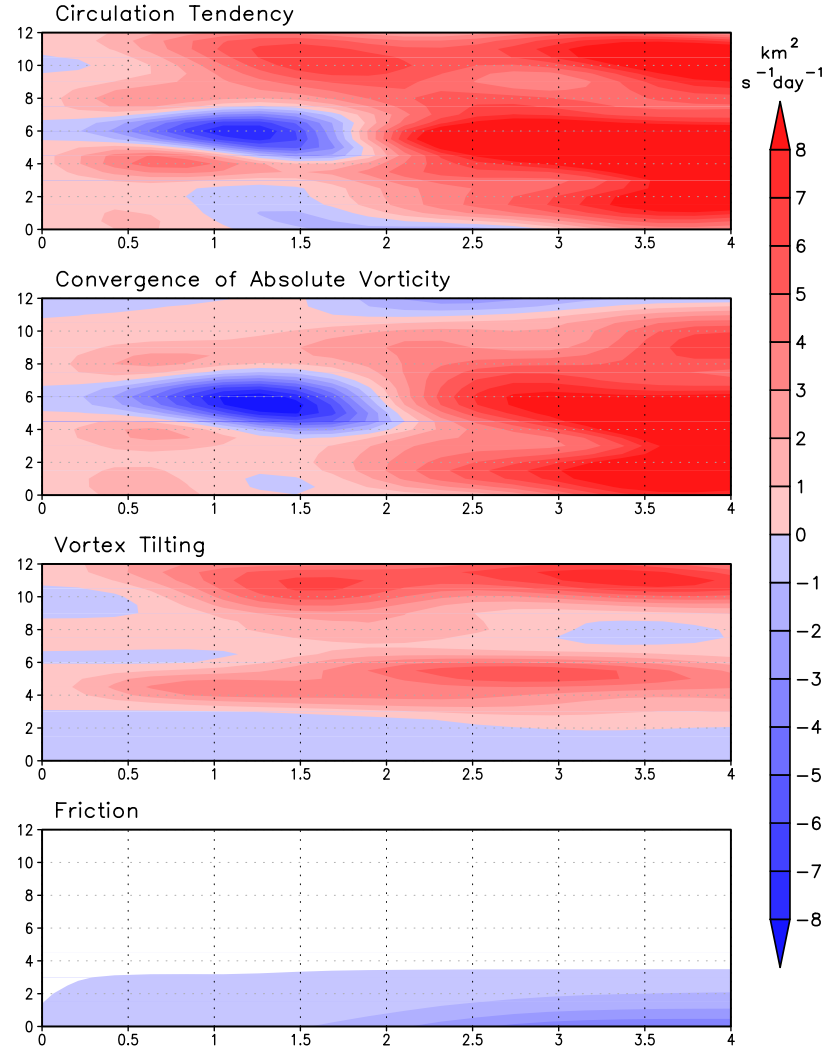

Figure 12. Four-panel height/distance cross section of each term from the circulation tendency equation (Eq. 7) for Nuri 1. The abscissa is the length of the box (degrees) on which the integration is performed and is centered on the $1.5 \mathrm{~km}$ sweet spot position. The ordinate is height $(\mathrm{km})$.

flectivity at mid-levels (Fig. 10). Infrared satellite imagery (not shown) verifies the presence of deep convection in these regions at these time periods (see Fig. 10, 00:00 UTC $16 \mathrm{Au}-$ gust, in Montgomery et al., 2010a). Co-location of the maxima in areas of low-level stretching tendency with deep convection suggests concentration of cyclonic vorticity through vortex tube stretching within the wave pouch. The dynamical processes observed here are consistent with the mechanisms necessary to develop a tropical cyclone strength vortex as outlined in DMW09 and illustrated by way of explicit numerical simulation in Montgomery et al. (2010b) and Wang et al. (2010a, b) at cloud-permitting scales.

To assess the possible organization and upscale vorticity growth near the sweet spot, we will examine a height/distance cross section of the frequency of occurrence of OW values greater than zero (Fig. 11). The thick black line highlights the $50 \%$ contour. Nuri 1 exhibits a layer of greater than $50 \%$ positive OW from the sweet spot out to approximately 1.5 degrees radius. This layer of positive OW extends vertically to $4 \mathrm{~km}$. Nuri 2 shows that while the radius of greater than $50 \%$ coverage of positive OW does not extend much farther outward from the sweet spot position, the 
depth of this positively dominated OW area now extends contiguously up to $7.5 \mathrm{~km}$. At radii within 0.6 degrees from the sweet spot position, the frequency of positive $\mathrm{OW}$ is greater than $80 \%$. These findings are consistent with the building of the cyclonic vorticity monolith near the sweet spot through (i) vorticity intensification near the sweet spot caused by vortex stretching associated with increased convection between Nuri 1 and 2; (ii) increasing longitudinal alignment of the circulation in the vertical; and (iii) aggregation of vorticity near the sweet spot in the low- and mid-levels (above $5 \mathrm{~km}$ ).

Let us now move back up in horizontal scale in order to assess the spin-up of the pre-Nuri disturbance on the mesoscale. Figure 12 shows the analyzed circulation tendency (Eq. 7) for Nuri 1 using the $10 \mathrm{~km}$ SAMURAI analysis around boxes of progressively increasing size in 0.2 degree length increments centered on the $1.5 \mathrm{~km}$ sweet spot position. The top row of Fig. 12 shows the net tendency of the absolute circulation during Nuri 1 over the analyzed area in a distance/height format. The next three rows show the individual terms comprising the circulation tendency equation (Eq. 7 ) in the same distance/height format. The results indicate

(i) Low-level (0 to $4 \mathrm{~km}$ altitude) spin-up tendency out to 0.8 degrees from the sweet spot. In this interior region, the spin-up is a result of the influx of absolute cyclonic vorticity dominating frictional spin-down.

(ii) Mid-tropospheric (4-7 km altitude) spin-down tendency from the sweet spot to approximately two degrees distance. Vortex misalignment plays a role in creating this area of mid-level spin-down tendency, as seen in the negative convergence tendency at mid-levels.

(iii) Barring a shallow region ( $<1 \mathrm{~km}$ depth) near the surface for 2 and 2.7 degree boxes around the sweet spot, there is a clear spin up tendency throughout the observed troposphere for boxes greater than 2 degree lengths.

(iv) Maximum frictional spin down tendency occurs in the boundary layer beyond 2.25 degree lengths.

(v) An area of low-level spin-down tendency located within the 1-2 degree boxes from the sweet spot (connecting to the aforementioned surface-based spin-down tendency).

While the surface-based spin-down found for the 2-2.7 degree lengths is primarily caused by frictional spin-down exceeding the influx of absolute vorticity, the region of spindown above the boundary layer between 1-2 degree box lengths is a combination of friction, negative eddy fluxes (not shown), and negative contributions from the tilting term. In summary: the foregoing analysis suggests that there is a combination of low-level spin-up (where convergence of absolute vorticity dominates frictional spin-down) and spin-down within the wave pouch. However, on the system scale, these data indicate that the pre-Nuri disturbance during the first day of observations is not spinning down, but broadly undergoing spin-up in the low levels.

\subsection{System-scale view of Nuri's early spin-up}

A complementary way to investigate whether the systemscale circulation is spinning up may be obtained by examining the time evolution of azimuthally averaged absolute angular momentum surfaces between Nuri 1 and 2 (e.g., Montgomery and Smith, 2014, their Fig. 13). In this paper, Fig. 13a, b shows radius-height cross sections of azimuthally averaged tangential wind (top, shaded) and absolute angular momentum (top, contours) for the Nuri 1 (a) and Nuri 2 (b) flights, respectively. Figure 13c (bottom) shows the difference in azimuthally averaged tangential wind between Nuri 2 and Nuri 1. The absolute angular momentum is defined by $M=r v+f r^{2} / 2$, where $r$ is the radius from the sweet spot position, $v$ is the azimuthally averaged, stormrelative tangential velocity, and $f$ is the Coriolis parameter. Above the frictional boundary layer, $M$ is approximately materially conserved. Therefore, as rings of fluid are drawn inwards, the corresponding tangential wind increases.

In the case of Nuri 1, the maximum tangential wind is in the lower levels and is located near the outermost ranges of the domain. For Nuri 2, the maximum low-level tangential wind has moved inward, closer to the sweet spot, and the magnitude of the tangential wind has increased in the lower and mid-troposphere. The increase of tangential wind in the mid-troposphere is consistent with an improved vertical alignment of the vortex as discussed above and suggests a deepening wave pouch.

The $M$ surfaces show higher values of $M$ moving inwards between Nuri 1 and 2. As discussed above, inward-moving $M$ surfaces, in conjunction with its approximate material conservation above the boundary layer, imply an amplification of the pouch-relative tangential wind field. These characteristics are consistent with a system undergoing development between Nuri 1 and 2 and affirm that, on the system scale, the pre-Nuri disturbance is broadly undergoing spinup in the low levels between Nuri 1 and 2 .

\section{Discussion: relation with prior work of RL11 and Raymond et al. (2011)}

The present kinematical and dynamical analysis of Nuri's genesis observed during the TCS-08 experiment has produced some very similar results to those found in RL11 and Raymond et al. (2011). In particular, we have found similar distributions of low- and mid-level vertical vorticity; similar areas of positive vortex stretching in the PBL and flow interior that are co-located with deep moist convection; a similar southeastward vortex tilt between the low- and mid-level mesoscale circulations; and a similar vertical wind shear pattern (not shown). The consistency of the major structural 


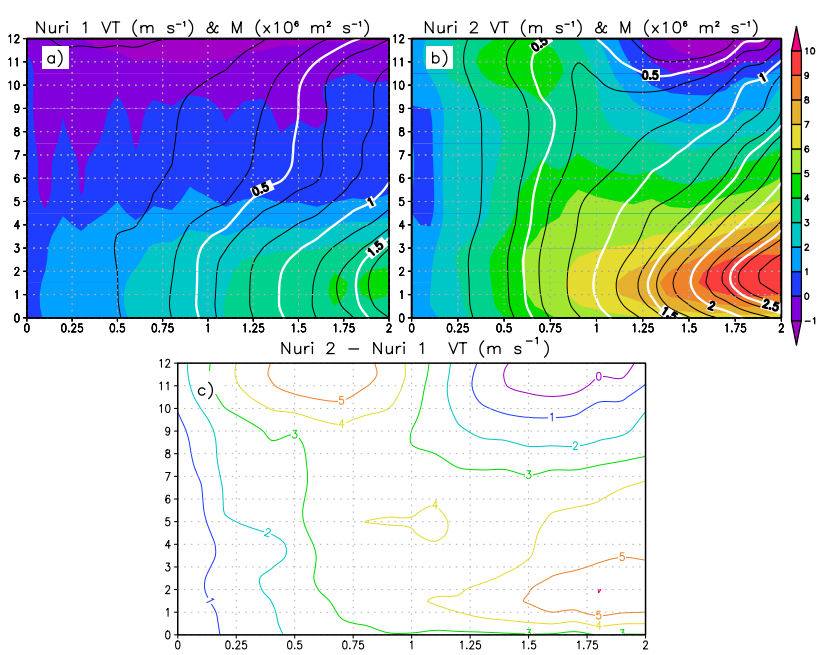

Figure 13. Three-panel plot of tangential wind (top, shaded) and absolute angular momentum (top, contours) for Nuri 1 (a) and Nuri 2 (b). Angular momentum surfaces are plotted at $0.2 \times 10^{6} \mathrm{~m}^{2} \mathrm{~s}^{-1}$ contours (black) and are highlighted (white) at $0.5 \times 10^{6} \mathrm{~m}^{2} \mathrm{~s}^{-1}$ intervals. The difference in tangential wind speed between Nuri 2 and Nuri 1 is plotted in (c). The abscissa is radial distance (degrees) from the $1.5 \mathrm{~km}$ sweet spot position. The ordinate is height $(\mathrm{km})$.

features of Nuri's genesis using independent analysis techniques and software suggest that our results are not overly sensitive to the analysis methodology.

The main difference between these studies is that RL11 show that the circulation tendency below approximately $1.25 \mathrm{~km}$ is negative and thus, they conclude that the preNuri circulation is decaying in the PBL during the Nuri 1 mission. On the other hand, our results indicate that the lowlevel circulation on the system scale is not spinning down at all radii ${ }^{8}$. In fact, a diagnosis of the circulation tendency during Nuri 1 at varying distances from the sweet spot center (as opposed to vertical profiles calculated within a single area as employed by RL11) shows that the pre-Nuri disturbance is already spinning-up in the lower levels (below $4 \mathrm{~km}$ altitude) at various radii from the sweet spot position. Moreover, be-

\footnotetext{
${ }^{8}$ Because the areas in which circulation tendency calculated herein and that presented in RL11 differ, the comparison between the two results is not direct. However, we have gone to great lengths to prove that both analysis schemes produce broadly similar results (see Sect. 3.2 and our response to an Interactive Comment). Additionally, one of the initial results we produced during the early stages in our mesoscale analysis of Nuri's spin-up sequence was an attempt to exactly replicate Fig. 16 from RL11 (not shown). These results are similar enough to give us confidence that comparisons between the circulation tendency analyses are appropriate. Thus, although the circulation tendency comparison may not be a precise "apples-to-apples" comparison, our methodology provides new and useful information that is important to understanding Nuri's genesis process, information which is omitted in RL11 and Raymond et al. (2011).
}
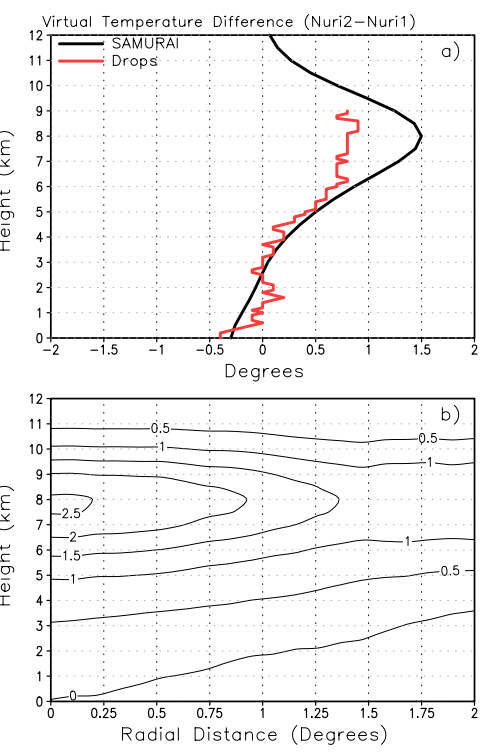

Figure 14. Vertical profile (a) of the mean virtual temperature difference over the observational domain from our SAMURAI analysis, black, and the dropwindsonde analysis of Montgomery and Smith (2012), red. Radius-height cross section (b) of the azimuthally averaged virtual temperature difference.

tween Nuri 1 and Nuri 2 we show that the low-level tangential wind increases at all radii from the sweet spot in both the dropwindsonde and SAMURAI analyses. The methodology of anchoring our analysis to the sweet spot position is optimally suited to observe and quantify the development that ensues around the translating sweet spot, the attractor point predicted by the new cyclogenesis model (Montgomery et al., 2012).

We now consider two additional quantities that will allow us to (i) further test the thermodynamic control hypothesis presented in Raymond et al. (2011) and RL11 and (ii) explain the differences in the findings they present compared to the findings presented herein.

\subsection{Mean virtual temperature profiles}

First, we examine thermodynamic data from the SAMURAI analysis in order to assess quantitatively the thermodynamic changes that have been hypothesized to be a critical influence on the spin-up process. Figure 14a is a vertical profile of the virtual temperature difference between Nuri 1 and 2, averaged over the observational domain. Aside from the smoothness of the SAMURAI-derived profile, on the pouch scale, there is little difference between the profile derived from the SAMURAI analysis and that derived by Montgomery and Smith (2012), who used only dropwindsonde data. These data suggest only a modest mid-tropospheric warming of 1 $\mathrm{K}$ and a very slight cooling $(<0.5 \mathrm{~K})$ in the troposphere below $2.75 \mathrm{~km}$. 
A more detailed look at the thermodynamic structure within the wave pouch may be obtained by examining the azimuthally averaged evolution of virtual temperature as a function of radial distance from the pouch center. This methodology allows for an assessment of whether there are regions within the wave pouch that exhibit increased thermal stabilization and permits one to test the statement by Raymond et al. (2011; p. 12) that the "... cold core at low levels was significantly enhanced in Nuri2 compared to Nuri1". Figure 14b shows a radius-height cross section of the change in virtual temperature from Nuri 1 to 2 . The figure shows that the strongest mid-level warming occurs near the center of the disturbance, and the low-level cooling is extremely weak (less than $0.5 \mathrm{~K}$ ), shallow, and confined largely to radii outside of 1 degree. In other words, we do not see evidence that the cold core was "significantly enhanced" between Nuri 1 and 2. Consistent with Montgomery and Smith (2012) and Smith and Montgomery (2012), we interpret the mid- and upper-level warming as simply the warm core of the developing tropical depression vortex via thermal wind balance. Qualitative consideration of thermal wind balance for a cyclonic vortex in gradient wind balance indeed affirms that the broad amplification of the low-level cyclonic winds in Fig. $13 \mathrm{c}$ is consistent with the warm-core thermal structure near the center of the pouch in Fig. 14b. These findings are consistent also with the numerical simulations of Wang (2012) who shows that the inner pouch region is characterized by mid-level warming and that weak cooling is found only at larger radii during the early tropical cyclone formation stage.

The foregoing findings are consistent with Montgomery and Smith (2012) in the sense that there is not a strong indication of the thermodynamic stabilization described by Raymond et al. (2011) on the pouch scale. Moreover, on the subpouch scale, the data does not suggest a significant enhancement of a cold core between Nuri 1 and 2 at low levels above the boundary layer ${ }^{9}$.

\subsection{Vertical mass flux profiles}

The second quantity we examine is the vertical mass flux ${ }^{10}$. Figure 15 shows the vertical mass flux profiles calculated

\footnotetext{
${ }^{9}$ Raymond et al. (2011) use saturated specific moist entropy as a proxy for temperature at a given altitude. Their Figs. 2 and 3 show a noticeable decrease in this quantity at the surface $\left(\sim 20 \mathrm{~J} \mathrm{~kg} \mathrm{~K}^{-1}\right)$ between Nuri 1 and 2. At the $0.625 \mathrm{~km}$ vertical level, however, their analysis suggests that the decrease in saturated-specific moist entropy is significantly smaller, less than $5 \mathrm{~J} \mathrm{~kg} \mathrm{~K}^{-1}$. Finally, between the $1.25 \mathrm{~km}$ and $5 \mathrm{~km}$ levels, there is no noticeable difference in saturated-specific moist entropy.

${ }^{10}$ For the vertical mass flux calculation, we reproduced the main results of RL11 using our independent 3-D-VAR analysis scheme. We integrated Equation 27 in RL11 over the observational area as defined in RL11 at each level to produce vertical mass flux profiles (Fig. 15).
}

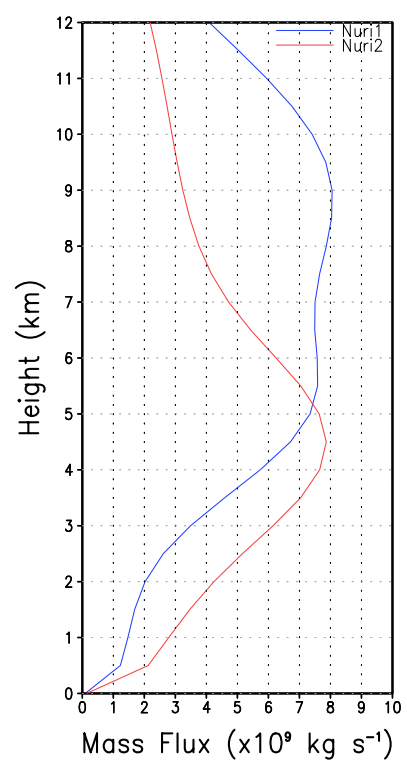

Figure 15. Vertical mass flux profiles calculated over the observational domain derived from the SAMURAI analysis for Nuri 1 (blue) and Nuri 2 (red).

over the observational domains. The mass flux profiles appear broadly similar to those presented in RL11, and confirm the lowering of the maximum vertical mass flux in the vertical from Nuri 1 to 2 . Now, the lowering of the mass flux profile is consistent with a change from a "top-heavy" latent heating profile (representative of a mixture of deep convection and stratiform convection processes, e.g., Houze 1989, 2004; Mapes and Houze 1995) to a profile more representative of deep convective processes. However, there are subtle differences between the RL11 mass flux profiles and those presented herein that invite careful examination. For Nuri 1, the mass flux in RL11 (their Fig. 16) is approximately zero from the surface to about $3 \mathrm{~km}$, and is negative in some of this vertical region. The RL11 analysis indicates also that the mass flux increases above $4 \mathrm{~km}$, with a maximum near 10 $11 \mathrm{~km}$. On the other hand, our Nuri 1 analysis suggests that the mass flux increases gradually from zero at the surface to $2 \mathrm{~km}$, followed by a more rapid increase until approximately $5 \mathrm{~km}$. Between $5-10 \mathrm{~km}$, there is a broad region of nearly constant mass flux, with an overall maximum near $9 \mathrm{~km}$, and a secondary maximum near $7.5 \mathrm{~km}$. For Nuri 2 , our mass flux profile displays an overall maximum near $4 \mathrm{~km}$, but decreases a bit more sharply above the $4 \mathrm{~km}$ maximum than the profile presented in RL11.

The quantitative differences highlighted above have important physical ramifications. Although both analyses have vertical mass flux maxima in the upper troposphere for Nuri 1, our analysis indicates positive mass flux values in the lower troposphere as well. Our analyses suggest also that the influence of the convective updrafts exceeds that of the stratiform downdrafts in the lower troposphere. In contrast, in the 
RL11 analysis the near zero lower tropospheric mass flux implies that on the system scale, convective updrafts are essentially balanced by downdrafts produced in the stratiform rain region. The positive low-level mass flux in our analysis ${ }^{11}$ is consistent with our dropwindsonde only and SAMURAI analyses, both of which indicate a spin-up of the lower and mid-troposphere from Nuri 1 to 2 .

Based on the foregoing results and discussion, the results presented herein suggest strongly that the observed protovortex is well into the cyclogenesis process, independent of the hypothesized 'thermodynamic control' as summarized in the Introduction. While we certainly recognize the importance of thermodynamics in the tropical cyclogenesis sequence (Smith and Montgomery, 2012; Montgomery and Smith, 2012), we believe that the dynamical and thermodynamic processes described in DMW09, Montgomery et al. (2010b), Wang et al. (2010a, b) and Wang (2012) lead naturally to low-level spin-up and vortex formation in the Kelvin cat's eye recirculation region of the tropical wave without the need for thermal stabilization associated with very small temperature anomalies ( $\sim 0.5 \mathrm{~K}$ or less $)$. To the extent that a small mid-level stabilization does occur outside the core of the pouch over the two days of intensive sampling of Nuri's genesis, our findings suggest that this stabilization is more incidental than essential. In other words, our analysis suggests that the proposed thermodynamic control presented by Raymond may not be a necessary condition for low-level spin-up in the case of Typhoon Nuri.

\section{Conclusions}

In this paper we have continued our study of the genesis of Typhoon Nuri (2008). Here we have examined the kinematics, dynamics, and thermodynamics of the genesis of Nuri using aircraft data from the USAF C130 and NRL P3 for two missions on 15-16 and 16-17 August. The mesoscale analysis presented herein is complimentary to the large-scale analysis presented by Montgomery et al. (2010a) and the thermodynamical analysis of Montgomery and Smith (2012).

During the first mission, the disturbance was a tropical easterly wave and prior to the second mission the disturbance had been upgraded to a TD. Visual inspection of the dropwindsonde data suggests that the disturbance-relative tangential wind speed increased in the low- and mid-levels between Nuri 1 and 2. Using dropwindsonde data, we have shown that the magnitude of the low-level (at and below $800 \mathrm{hPa}$ ) tangential winds did increase from Nuri 1 to 2 at all radii within

\footnotetext{
${ }^{11}$ It is a useful reminder to recall that the low-level spin-up discussed herein is caused by the system-scale convergence of vorticity substance induced by the aggregate of deep convective clouds in the pouch and the inflow generated thereto. The area-averaged vertical mass flux profile is not the cause of the spin-up. Rather, it is simply an indicator of the nature of the precipitation on the pouch scale.
}

the wave pouch. These findings indicate that there was lowlevel spin-up occurring between Nuri 1 to 2 .

To compliment the dropwindsonde-only results, analyses from a newly developed variational scheme that synthesizes aircraft radar and dropwindsonde data are presented. Stormrelative circulation tendency, absolute angular momentum, and tangential wind are computed at various distances and heights from the sweet spot center throughout the observed domain for each flight. The advantages of performing such calculations at several radii from the sweet spot position include the ability to examine the dynamical processes at all observed distances within the wave pouch and the ability to directly compare data from multiple research flights that may have different areas of data collection. Results from the variational analysis are consistent with the dropwindsonde-only analysis in that they indicate that the low- and mid-level tangential winds increased in magnitude and the tangential wind maximum moved inwards from Nuri 1 to 2 . These results are consistent with the vorticity aggregation process. Additionally, by examining these data in this fashion, we are able to observe and quantify the role of vertical alignment in the building of the cyclonic vorticity monolith.

By calculating the circulation tendency for Nuri 1, we have identified regions indicating both low-level spin-up and spindown within the wave pouch. Further examination indicates that the low-level spin-down results from a combination of frictional spin-down, negative vortex tilting, and eddy flux divergence out of the integration area. There were areas of low-level spin-up located closest to the sweet spot position.

From a vorticity perspective, vorticity is observed to amplify most significantly near the sweet spot position, initially at the lower levels and increasing in intensity with time. Areas of positive vortex stretching in the lower troposphere associated with moist convection lead to vorticity intensification in the lower levels within the pouch and suggest the presence of rotating deep convection. The regions of vortextube stretching increased in areal coverage and magnitude between Nuri 1 and 2 . All of these findings are consistent with those of RL11.

On the system scale, inward movement of absolute angular momentum surfaces confirms the convergence of absolute angular momentum, which is one of two system-scale mechanisms responsible for tropical cyclone spin-up (Smith et al., 2009; Montgomery and Smith, 2014). Radial-height profiles derived from the SAMURAI analysis show that the maximum increase in tangential winds is found to have occurred in the low levels near the top of the boundary layer. This increase is consistent with the hypothesis of a progressive boundary layer control during the spin-up process (Montgomery and Smith, 2011). In short, the foregoing properties are consistent with those of an intensifying proto-vortex from the beginning of the observation period.

The analyses and interpretations presented herein have provided answers to the three questions posed in the Introduction. First, the magnitude of the low- and mid-level 
circulation increased from Nuri 1 to 2. Second, for the case of Typhoon Nuri, our analysis suggests that any thermal stabilization of the atmosphere occurring during Nuri 2 appears incidental rather than essential to the storm development. Third, the distinct advantages of examining the dynamics of tropical cyclogenesis in a frame of reference anchored on the translating sweet spot position were demonstrated for these observational analyses.

The findings of this study are consistent in some respects to that of Raymond and colleagues, but differ in their suggested key result and related scientific implication that the pre-Nuri disturbance was spinning down in the boundary layer on the first day of observations. The findings herein support the marsupial model positing that the Kelvin cat's eye circulation of the parent wave-like disturbance provides a favorable environment for intrinsic convective vorticity organization and low-level spin-up on the mesoscale. The findings further support the applicability of the marsupial paradigm for easterly wave-like disturbances in the western North Pacific basin.

Acknowledgements. Lou Lussier acknowledges the support from the National Research Council (NRC), through its Research Associateship Program, and the host institution, the Naval Postgraduate School (NPS) in Monterey, California. The work of all authors was partially supported by the Office of Naval Research (ONR), through award N0001411WX20095, and by the National Science Foundation (NSF), through awards AGS 0733380 and 0851077. The ELDORA and dropwindsonde data for this study were provided by NCAR/EOL under sponsorship of the National Science Foundation.

Edited by: T. J. Dunkerton

\section{References}

AMS Statement: Hurricane forecasting in the United States, B. Am. Meteorol. Soc., 88, 950-951, 2007.

Bell, M. M., Montgomery, M. T., and Emanuel, K. A.: Air-sea enthalpy and momentum exchange at major hurricane wind speeds observed during CBLAST, J. Atmos. Sci., 69, 3197-3222, 2012.

Bell, M. M., Lee, W.-C., Wolff, C., and Cai, H.: A Solo-based automated quality control algorithm for airborne Doppler tail radar data, J. Appl. Meteor. Clim., 52, 2509-2528, 2013.

Chang, C. P., Morris, V. F., and Wallace, J. M.: A statistical study of easterly waves in the western Pacific: July-December 1964, J. Atmos. Sci., 27, 195-201, 1970.

Davis, C. A. and Ahijevych, D. A.: Mesoscale structural evolution of three tropical weather systems observed during PREDICT, J. Atmos. Sci., 69, 1284-1305, 2012.

Davis, C. A. and Galarneau Jr, T. J.: The vertical structure of mesoscale convective vortices, J. Atmos. Sci., 66, 686-704, 2009.

Dunkerton, T. J., Montgomery, M. T., and Wang, Z.: Tropical cyclogenesis in a tropical wave critical layer: easterly waves, Atmos. Chem. Phys., 9, 5587-5646, doi:10.5194/acp-9-5587-2009, 2009.
Elsberry, R. L. and Harr, P. A.: Tropical Cyclone Structure (TCS08) field experiment science basis, observational platforms, and strategy, Asia-Pac. J. Atmos. Sci., 44, 209-231, 2008.

Haynes, P. H. and McIntyre, M. E.: On the evolution of vorticity and potential vorticity in the presence of diabatic heating and frictional or other forces, J. Atmos. Sci., 44, 828-841, 1987.

Houze, R. A.: Observed structure of mesoscale convective systems and implications for large-scale heating, Q. J. Roy. Meteorol. Soc., 115, 425-461, 1989.

Houze Jr, R. A.: Mesoscale convective systems, Rev. Geophys., 42, RG4003, doi:10.1029/2004RG000150, 2004.

López Carrillo, C. and Raymond, D. J.: Retrieval of threedimensional wind fields from Doppler radar data using an efficient two-step approach, Atmos. Meas. Tech., 4, 2717-2733, doi:10.5194/amt-4-2717-2011, 2011.

Lussier III, L. L.: A multi-scale analysis of tropical cyclogenesis within the critical layer of tropical easterly waves within the Atlantic and western North Pacific sectors, PhD Dissertation, Naval Postgraduate School, Monterey, CA, 2010.

Mapes, B. E. and Houze Jr., R. A.: Diabatic divergence profiles in western Pacific mesoscale convective systems, J. Atmos. Sci., 52, 1807-1828, 1995.

Marín, J. C., Raymond, D. J., and Raga, G. B.: Intensification of tropical cyclones in the GFS model, Atmos. Chem. Phys., 9, 1407-1417, doi:10.5194/acp-9-1407-2009, 2009.

McWilliams, J. C.: The emergence of isolated coherent vortices in turbulent flow, J. Fluid Mech., 140, 21-43, 1984.

Montgomery, M. T. and Smith, R. K.: Tropical cyclone formation: Theory and idealized modeling. In Proceedings of Seventh WMO International Workshop on Tropical Cyclones (IWTCVII), La Réunion, November 2010. (WWRP 2011-1) World Meteorological Organization: Geneva, Switzerland, 2011.

Montgomery, M. T. and Smith, R. K.: The genesis of Typhoon Nuri as observed during the Tropical Cyclone Structure 2008 (TCS08) field experiment - Part 2: Observations of the convective environment, Atmos. Chem. Phys., 12, 4001-4009, doi:10.5194/acp-124001-2012, 2012.

Montgomery, M. T. and Smith, R. K.: Paradigms for tropical cyclone intensification, Austr. Meteor. Oceanogr. J., in press, 2014.

Montgomery, M. T., Lussier III, L. L., Moore, R. W., and Wang, Z.: The genesis of Typhoon Nuri as observed during the Tropical Cyclone Structure 2008 (TCS-08) field experiment - Part 1: The role of the easterly wave critical layer, Atmos. Chem. Phys., 10, 9879-9900, doi:10.5194/acp-10-9879-2010, 2010a.

Montgomery, M. T., Wang, Z., and Dunkerton, T. J.: Coarse, intermediate and high resolution numerical simulations of the transition of a tropical wave critical layer to a tropical storm, Atmos. Chem. Phys., 10, 10803-10827, doi:10.5194/acp-1010803-2010, 2010b.

Montgomery, M. T., Davis, C., Dunkerton, T., Wang, Z., Velden, C., Torn, R., Majumdar, S. J., Zhang, F., Smith, R. K., Bosart, L., Bell, M. M., Haase, J. S., Heymsfield, A., Jensen, J., Campos, T., and Boothe, M. A.: The Pre-Depression Investigation of Cloudsystems in the Tropics (PREDICT) Experiment, 93, B. Am. Meteorol. Soc., 153-172, 2012.

Raymond, D. J. and López Carrillo, C.: The vorticity budget of developing typhoon Nuri (2008), Atmos. Chem. Phys., 11, 147163, doi:10.5194/acp-11-147-2011, 2011. 
Raymond, D. J. and Sessions, S. L.: Evolution of convection during tropical cyclogenesis, Geophys. Res. Lett., 34, L06811, doi:10.1029/2006GL028607, 2007.

Raymond, D. J., Sessions, S. L., and López-Carrillo, C.: Thermodynamics of tropical cyclogenesis in the Northwest Pacific, J. Geophys. Res., 116, D18101, doi:10.1029/2011JD015624, 2011.

Reed, R. J. and Recker, E. E.: Structure and properties of synopticscale wave disturbances in the equatorial western Pacific, J. Atmos. Sci., 28, 1117-1133, 1971.

Rutherford, B. and Montgomery, M. T.: A Lagrangian analysis of a developing and non-developing disturbance observed during the PREDICT experiment, Atmos. Chem. Phys., 12, 11355-11381, doi:10.5194/acp-11-11355-2011, 2011.

Sanger, N. T., Montgomery, T. M., Smith, R. K., and Bell, M. M.: An Observational Study of Tropical Cyclone Spinup in Supertyphoon Jangmi (2008) from 24 to 27 September, Mon. Weather Rev., 142, 3-28, 2014.

Schubert W. H. and Alworth, B. T.: Evolution of potential vorticity in tropical cyclones, Q. J. Roy. Meteorol. Soc., 113, 147-162, 1987.

Smith, R. K. and Montgomery, M. T.: Observations of the convective environment in developing and non-developing tropical disturbances, Q. J. Roy. Meteorol. Soc., 138, 1721-1739, 2012.
Smith, R. K., Montgomery, M. T., Sang, N. V.: Tropical cyclone spin-up revisited, Q. J. Roy. Meteorol. Soc., 135, 1321-1335, 2009.

Wang, Z.: Thermodynamic Aspects of Tropical Cyclone Formation, J. Atmos. Sci., 69, 2433-2451, 2012.

Wang, Z., Montgomery, M. T., and Dunkerton, T. J.: A dynamicallybased method for forecasting tropical cyclogenesis location in the Atlantic sector using global model products, Geophys. Res. Lett., 36, L03801, doi:10.1029/2008GL035586, 2009.

Wang, Z., Montgomery, M. T., and Dunkerton, T. J.: Genesis of pre-hurricane Felix (2007) Part I: The role of the easterly wave critical layer, J. Atmos. Sci., 67, 1711-1729, 2010a.

Wang, Z., Montgomery, M. T., and Dunkerton, T. J.: Genesis of prehurricane Felix (2007) Part II: Warm core formation, precipitation evolution and predictability. J. Atmos. Sci., 67, 1730-1744, 2010b.

Weiss, J.: The dynamics of enstrophy transfer in two-dimensional hydrodynamics, Physica D: Nonlinear Phenomena, 48 273-294, 1991.

Willoughby H. E.: Mature structure and evolution. Global Perspectives on Tropical Cyclones. WMO/TD-No. 693, edited by: Elsberry R. L., World Meteorological Organization: Geneva, 21-62, 1995. 